\title{
Complete Classification and Efficient Determination of Arrangements Formed by Two Ellipsoids
}

\author{
XIAOHONG JIA, KLMM, AMSS, Chinese Academy of Sciences \& University of Chinese Academy of Science, China \\ CHANGHE TU, School of Computer Science and Technology, Shandong University, China \\ BERNARD MOURRAIN, Aromath, Inria Méditerranée, Université Côte d'Azur, France \\ WENPING WANG, The University of Hong Kong, Hong Kong
}

\begin{abstract}
Arrangements of geometric objects refer to the spatial partitions formed by the objects and they serve as an underlining structure of motion design, analysis and planning in CAD/CAM, robotics, molecular modeling, manufacturing and computer-assisted radiosurgery. Arrangements are especially useful to collision detection, which is a key task in various applications such as computer animation, virtual reality, computer games, robotics, CAD/CAM and computational physics.

Ellipsoids are commonly used as bounding volumes in approximating complex geometric objects in collision detection. In this paper we present an in-depth study on the arrangements formed by two ellipsoids. Specifically, we present a classification of these arrangements and propose an efficient algorithm for determining the arrangement formed by any particular pair of ellipsoids. A stratification diagram is also established to show the connections among all the arrangements formed by two ellipsoids. Our results for the first time elucidate all possible relative positions between two arbitrary ellipsoids and provides an efficient and robust algorithm for determining the relative position of any two given ellipsoids, therefore providing the necessary foundation for developing practical and trust-worthy methods for processing ellipsoids for collision analysis or simulation in various applications.
\end{abstract}

Additional Key Words and Phrases: Arrangements, Ellipsoids, Collision Detection

\section{ACM Reference Format:}

Xiaohong Jia, Changhe Tu, Bernard Mourrain, and Wenping Wang. 2020. Complete Classification and Efficient Determination of Arrangements Formed by Two Ellipsoids. ACM Trans. Graph. 39, 3, Article 27 (May 2020), 13 pages. https://doi.org/10.1145/nnnnnnn. nnnnnnn

\section{INTRODUCTION}

The arrangement $\mathcal{A}(\mathcal{S})$ of a given collection $\mathcal{S}$ of geometric objects in $\mathbb{R}^{d}$ is the decomposition of $\mathbb{R}^{d}$ into connected open cells of $0,1, \cdots, d$ dimensions induced by $\mathcal{S}$. There is rich literature on the arrangements of lines and hyperplanes in both

Authors' addresses: Xiaohong Jia, KLMM, AMSS, Chinese Academy of Sciences \& University of Chinese Academy of Science, Beijing, 100190 China, xhjia@amss.ac.cn; Changhe Tu, School of Computer Science and Technology, Shandong University, Qingdao, 266237, China, chtu@sdu. edu.cn; Bernard Mourrain, Aromath, Inria Méditerranée, Université Côte d'Azur, 06902, France, Bernard.Mourrain@inria.fr; Wenping Wang, The University of Hong Kong, Department of Computer Science, Hong kong, Hong Kong, wenping@cs.hku.hk.

(C) 2020 Association for Computing Machinery.

This is the author's version of the work. It is posted here for your personal use. Not for redistribution. The definitive Version of Record was published in ACM Transactions on Graphics, https://doi.org/10. 1145/nnnnnnn.nnnnnnn. combinatorial and computational geometry [Berg et al. 2013; Dimca 2017; Felsner 2004; Halperin 2004]. Arrangements of geometric objects are used as an underlining structure of motion design, analysis and planning in CAD/CAM, robotics, molecular modeling, manufacturing as well as computerassisted radio-surgery [Agarwal et al. 1998; Halperin 2004; Halperin and Shelton 1997; Schweikard et al. 1992].

An important application of arrangements is collision detection, a key task in various applications such as computer animation, virtual reality, computer games, robotics, CAD/CAM, and computational physics [Eberly 2001; Gottschalka et al. 1996; Hubbard 1996; Mirtich 1998; Palmer and Grimsdale 1995; Tang et al. 2014; Wang 2014]. Because direct collision detection by complex objects is usually very time-consuming, a typical approach of simplification is to approximate the complex objects by bounding volumes.

Two criteria are usually taken into consideration in choosing the type of bounding volumes: the bounding tightness in approximating the complex objects and the availability of an efficient algorithm for detecting the collision between the type of bounding volumes to be adopted. Simple geometric primitives such as oriented bounding boxes, spheres and discrete oriented polytopes have frequently been used as bounding volumes [Chang et al. 2008; Gottschalka et al. 1996; Hubbard 1996; Klosowski et al. 2002].

Ellipsoids have superior capability of shape approximation and relatively low algebraic degree, hence have widely been used as bounding volumes in various applications, such as computer vision [Liang et al. 2016], robotics [Lee et al. 2017], mechanism and machine theory [Goncalves et al. 2017], molecular dynamics [Ghossein and Lévesque 2013], computational particle mechanics [Rubio-Largo et al. 2015] as well as in material science [Xu and Chen 2016]. See Fig. 1 for illustrations of ellipsoidal bounding volumes in different applications. Despite ellipsoids may serve as much tighter bounding primitives than spheres, currently ellipsoids are not used as commonly as spheres as approximate shape proxies in collision detection and simulation. This is largely due to the lack of theoretical understanding and efficient algorithms for handling ellipsoid$\mathrm{s}$, which provides the motivation of our present work. By providing comprehensive analysis of the relationship of two ellipsoids and provide efficient methods for the classification, our work aims to tackle this problem and to inspire more follow-up research on the efficient use of ellipsoids in various fields. In fact, efficient methods for processing ellipsoids are 
in great demand since ellipsoids are already used as bounding volumes or shape proxies in a number of fields as listed above.

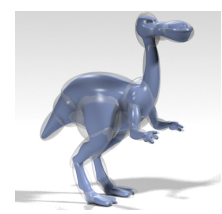

(a)

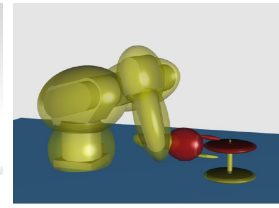

(b)

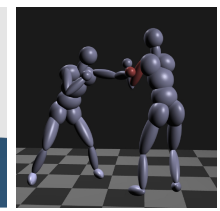

(c)
Fig. 1. Ellipsoidal Bounding Volumes. Courtesy of [Lu et al. 2007] for (a), [Choi 2008] for (b), [Choi et al. 2009] for (c).

In some applications we are not only interested in the separation of two closed volumes (such as collision detection) but also concerned about the containment or other general and more complex relationship of two objects. For example, one ellipsoid may move while keeping tangential contact, externally or internally, with another static ellipsoid. The arrangements of spheres or ellipsoids have been applied in computer-assisted radio-surgery [Schweikard et al. 1992], molecular modeling [Halperin and Shelton 1997], assembling planning [Halperin 2004] and manufacturing [Agarwal et al. 1998]. However, to our knowledge, there is no previous study on the full classification of the arrangements of ellipsoids and their fast computation.

In this paper, we will present an in-depth and systematic study on the arrangements of a pair of ellipsoids. Our treatment will be finer than the usual definition of arrangements besides the different decompositions of 3D space, we also take the degeneracy of the intersection curve of the two ellipsoids into consideration, including the existence and different types of singularities (cusp, crunode or acnode) on the intersection curve.

Our contributions are as follows.

(1) (Enumeration) A full enumeration of 21 non-equivalent classes of the arrangements of a pair of ellipsoids is given;

(2) (Determination) An algorithm is designed to determine the arrangement for a given pair of ellipsoids;

(3) (Stratification) A connection diagram is constructed for all the 21 arrangement classes, to show whether any two classes can transit into each other directly without going through another intermediate class under continuous motions and deformations of the two ellipsoids.

These results elucidate all possible relative positions between two arbitrary ellipsoids and provides an efficient and robust algorithm for determining the relative position of any two given ellipsoids, therefore providing the necessary foundation for developing practical and trust-worthy methods for processing ellipsoids for collision analysis or simulation in various applications.

\section{RELATED WORKS}

\subsection{Arrangements}

Arrangements have been extensively studied in both combinatorial and computational geometry. Results on arrangements in combinatorial geometry can be found in [Felsner 2004]. An early survey of research on arrangements from the viewpoint of computational geometry is given by [Halperin and Shelton 1997]. Readers are also referred to [Berg et al. 2013; Halperin 2004] and [Dimca 2017] for the basic concepts, problems and applications related to arrangements of lines, hyperplanes and curved surfaces. Many developments in the research on arrangements have been motivated by problems related to robot motion planning; see chapter 47 and chapter 48 in [Halperin 2004]. Arrangements of spheres have also found their wide applications in computer-assisted radio-surgery [Schweikard et al. 1992], molecular modeling [Halperin and Shelton 1997], assembling planning [Halperin 2004] and manufacturing [Agarwal et al. 1998]. Arrangements are also used for solving problems in geometric optimization, range searching, statistical analysis, and micro robotics [Agarwal et al. 1998; Halperin 2004].

\subsection{Ellipsoids}

Ellipsoids have widely been used as bounding volumes in various applications, such as computer vision [Liang et al. 2016], robotics [Lee et al. 2017], mechanism and machine theory [Goncalves et al. 2017], molecular dynamics [Ghossein and Lévesque 2013], computational particle mechanics [RubioLargo et al. 2015] as well as in material science [Xu and Chen 2016]. In particular, ellipsoids have been a popular candidate for bounding volumes in collision detection; see for examples [Choi et al. 2009, 2006; Jia et al. 2011; Ju et al. 2001; Liu et al. 2007; Lu et al. 2007; Rimon and Boyd 1977; Shiang et al. 2000; Wang et al. 2004].

A simple algebraic condition is given by [Wang et al. 2001] on deciding the basic relative positions, i.e., separation, overlapping or containment of two ellipsoids. This condition is translated in [Jia et al. 2011] in terms of more elementary computations which apply to continuous collision detection of two moving ellipsoids. Similar problem of characterizing the relative position of two ellipses has been considered by [AlberichCarraminana et al. 2017; Etayo et al. 2006], where algebraic conditions are used.

Ellipsoids are a subset of quadrics, which are well explored in geometric modeling. See [Wang 2002] for an overview of quadrics in both their algebraic background and various applications in geometric modeling. Much attention has been paid to the intersection curve of two quadrics. Existing works can be classified to two categories: geometric approaches that serve for tracing the curve for graphical display use [Miller 1987; Miller and Goldman 1995; Shene and Johnstone 2014]; and the algebraic approaches that focus on exact parametric representation [Dupont et al. 2008a,b,c; Levin 1979] or full classifications of the morphologies of the intersection curve of two quadrics [Bromwich 1960; Farouki et al. 1989; Tu et al. 
2009]. The Segre characteristic serves as the most important algebraic ingredient in [Bromwich 1960; Farouki et al. 1989; Tu et al. 2009], and it also casts light on our exploration in arrangements of ellipsoid pairs.

\section{ARRANGEMENTS}

An ellipsoid is represented by $\mathcal{A}: X^{T} A X=0$ where $X=$ $(x, y, z, 1)^{T}$ are homogeneous coordinates of a point in $\mathbb{R}^{3}$, and $A$ a $4 \times 4$ real symmetric matrix. We assume that the matrix $A$ is normalized so that $X_{0}^{T} A X_{0}<0$ for a point $X_{0}$ interior to $\mathcal{A}$.

An arrangement of a pair of ellipsoid is a decomposition of the 3D space by the two ellipsoids. Fig. 2 shows four arrangements of two ellipsoids, among many that are possible.

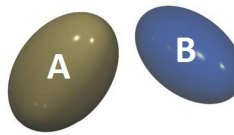

(a)

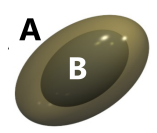

(b)

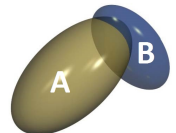

(c)

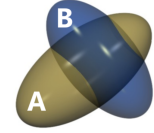

(d)
Fig. 2. Four arrangements of two ellipsoids

When the two ellipsoids can move or deform continuously, as long as they do not change their way of decomposing the space (in the homomorphism sense), we consider the type of the resulting arrangement to remain unchanged, i.e. equivalent. See Fig. 3 for two pairs of equivalent arrangements. A formal definition of the equivalence classes of arrangements of two ellipsoids will be given shortly.

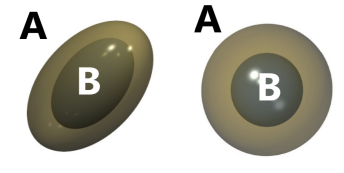

(a)

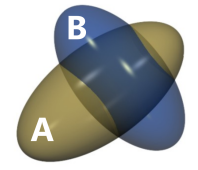

(b)

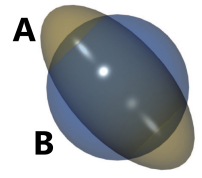

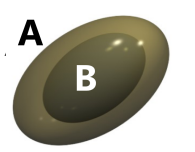

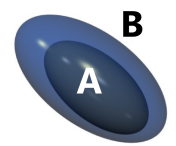

(a)

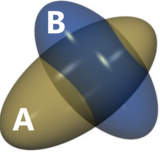

(b)

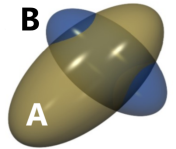

Fig. 4. Each pair of ellipsoids in (a) and (b) are not equivalent.

Now we are going to formally define the arrangements that can be formed by two arbitrary ellipsoids and their equivalent classes. Let us label the interior, exterior and the boundary of an ellipsoid $\mathcal{A}$ by $-1,1$, and 0 , respectively. Denote $W=\{-1,1,0\}$. Define the map $g_{A}: \mathbb{R}^{3} \rightarrow W$ by $g_{A}(X)=\operatorname{sign}\left(X^{T} A X\right)$. The space $\mathbb{R}^{3}$ defined by a pair of two ellipsoids $\mathcal{A}: X^{T} A X=0$ and $\mathcal{B}: X^{T} B X=0$ is divided into several parts, which is defined by

$$
\mathcal{M}_{A, B}(w)=\left\{X \in \mathbb{R}^{3} \mid g_{A, B}(X)=w\right\} \text { for } w \in W^{2},
$$

where $g_{A, B}(X)=\left(g_{A}(X), g_{B}(X)\right) \in W^{2}$ is the label of a point $X \in R^{3}$ with respect to the ellipsoids $\mathcal{A}$ and $\mathcal{B}, X \in \mathbb{R}^{3}$. The arrangement formed by the ellipsoids $\mathcal{A}$ and $\mathcal{B}$ is

$$
\mathcal{A R}(\mathcal{A}, \mathcal{B})=\left\{\left(\mathcal{M}_{A, B}(w), w\right), w \in W^{2}\right\} .
$$

For easy visualization, as illustration, the labels of points in $2 \mathrm{D}$ plane partitioned by two ellipses is shown by Fig. 5 . The partition of $3 \mathrm{D}$ space by two ellipsoids is an analogue to the $2 \mathrm{D}$ case.

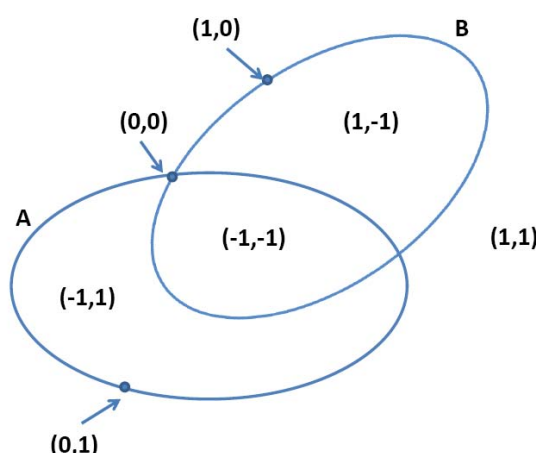

$(0,1)$

Fig. 5. The labels of $2 \mathrm{D}$ plane induced by the arrangement of two ellipses $\mathcal{A}$ and $\mathcal{B}$.

We shall also take the degeneracy of the intersection of the two ellipsoids into consideration when classifying their arrangements. For example, in Fig. 6(a), the two pairs of ellipsoids partition the space in a similar way, but the intersection curve for the pair on the right contains a crunode; in Fig. 6(b), the two pairs of ellipsoids partition the space in a similar way, but the intersection curve for the pair on the right contains a cusp. Hence we consider Fig. 6(a) and Fig. $6(\mathrm{~b})$ as two pairs of non-equivalent arrangements. 


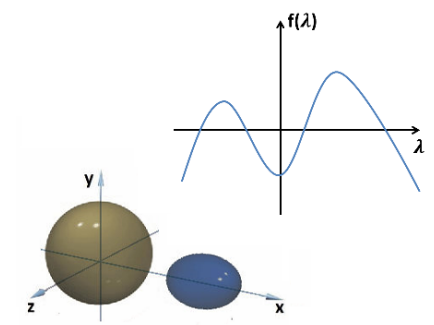

(a)

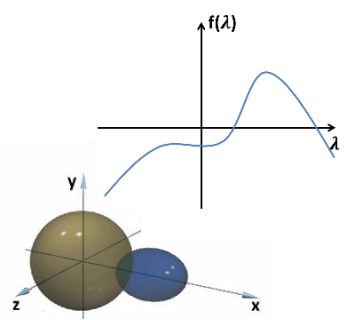

(b)

Fig. 7. The separation and overlapping of two ellipsoids are determined by the root pattern of the characteristic polynomial [Wang et al. 2001]. The two ellipsoids are (a). separated if and only if $f(\lambda)=0$ has two distinct real negative roots; and (b). overlapping if and only if $f(\lambda)=0$ has no real negative root.

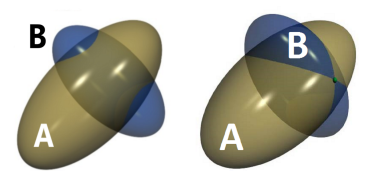

(a)

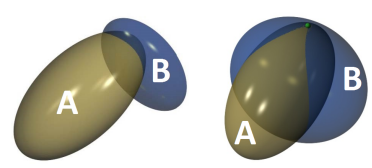

(b)

Fig. 6. Each pair of ellipsoids in (a) and (b) are not equivalent.

Therefore, two arrangements are said to be equivalent if there is a diffeomorphism $h(X)$ of $\mathbb{C}^{\infty}$ from $\mathbb{R}^{3}$ to itself such that any point $X$ and its image $h(X)$ has the same label.

\section{ALGEBRAIC CHARACTERIZATIONS}

\subsection{Characteristic polynomial}

A pair of ellipsoids $\mathcal{A}$ and $\mathcal{B}$ defines a quadric pencil $\lambda A-B$. The characteristic polynomial of the quadric pencil is defined as

$$
f(\lambda)=\operatorname{det}(\lambda A-B)
$$

which is a degree four polynomial in $\lambda$. A correspondence between the root pattern of $f(\lambda)=0$ and the basic relative position, i.e., separation or overlapping, of the two ellipsoids is essentially given by [Wang et al. 2001] ${ }^{1}$ as follows, illustrated in Fig. 7.

Lemma 4.1. [Wang et al. 2001] For two ellipsoids, $f(\lambda)=0$ has always two positive roots, counting multiplicities. Moreover, The two ellipsoids are separated/externally touching/ overlapping if and only if $f(\lambda)$ has two distinct negative roots/one double negative root/no negative root.

By Lemma 4.1, one can decide the basic relative positions, i.e., separation, externally touching and overlapping, of two ellipsoids by the real root pattern of the characteristic polynomial of the quadric pencil formed by these two ellipsoids.

${ }^{1}$ The characteristic equation in [Wang et al. 2001] takes the different
form of $f(\lambda)=\operatorname{det}(\lambda A+B)$. Therefore, it is the positive roots that are of concern there for detecting the separation of the two ellipsoids.

However, the root pattern of the characteristic polynomial is not enough to characterize the arrangement of two ellipsoids. For example, Fig. 8 shows three pairs of ellipsoids whose characteristic polynomials have the same root pattern of four different real positive roots. However, the arrangements of these three pairs of ellipsoids are non-equivalent to each other.

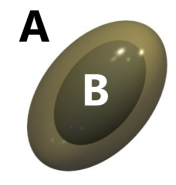

(a)

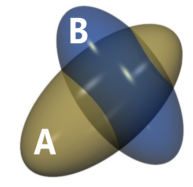

(b)

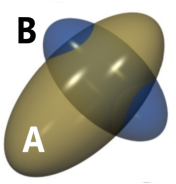

(c)
Fig. 8. Three non-equivalent arrangements. The characteristic polynomials of the three pairs of ellipsoids have the same root pattern of four real positive roots.

\subsection{Index sequence}

$\mathrm{Tu}$ et al. used a more in-depth algebraic characterization, index sequence, to classify the morphology of the intersection of two quadratic surfaces in $\mathbb{P R}^{3}$, the $3 \mathrm{D}$ real projective space [Tu et al. 2009]. The index sequence of a quadric pencil not only includes the root pattern of the characteristic polynomial, but also involves the Jordan form associated to each root and the information between two consecutive roots. To define index sequence, we need first to define the index function of a quadric pencil.

The index function of the quadric pencil formed by two quadrics $\mathcal{A}$ and $\mathcal{B}$ is defined as

$$
\operatorname{Id}(\lambda)=\operatorname{Index}(\lambda A-B), \lambda \in \mathbb{P R}
$$

where Index means the number of positive eigenvalues of a matrix. Since matrices $A$ and $B$ are both $4 \times 4$ matrices, $\operatorname{Id}(\lambda) \in\{0,1,2,3,4\}$.

The behavior of the index function for a pencil of quadrics is captured by the eigenvalue curve $\mathcal{C}$, defined by the equation

$$
C(\lambda, \mu)=\operatorname{det}(\lambda A-B-\mu I)=0
$$

in the $\lambda$ - $\mu$ plane. Note that $C$ has degree four in both $\lambda$ and $\mu$. Because $\lambda A-B$ is real symmetric, for each value of $\lambda \in \mathbb{R}$, there are in total four real roots for $C=0$ in $\mu$, counting multiplicities. Therefore, the curve $\mathcal{C}$ has four $\lambda$-monotone branches. For each value $\lambda_{0}$, the index function $\operatorname{Id}\left(\lambda_{0}\right)$ equals to the number of points of $\mathcal{C}$ on the vertical line $\lambda=\lambda_{0}$ and above the horizontal axis $\mu=0$. Fig. 9 shows the eigenvalue curve of a pair of ellipsoids, where $\operatorname{Id}\left(\lambda_{0}\right)=1, \operatorname{Id}\left(\lambda_{1}\right)=2$ and $\operatorname{Id}\left(\lambda_{2}\right)=3$. 
Fig. 9. The eigenvalue curve of two ellipsoids $\mathcal{A}: x^{2}+y^{2}+z^{2}-1=0$ and $\mathcal{B}: x^{2}+\frac{5}{2} y^{2}+3 z^{2}-y-\frac{3}{2}=0$.

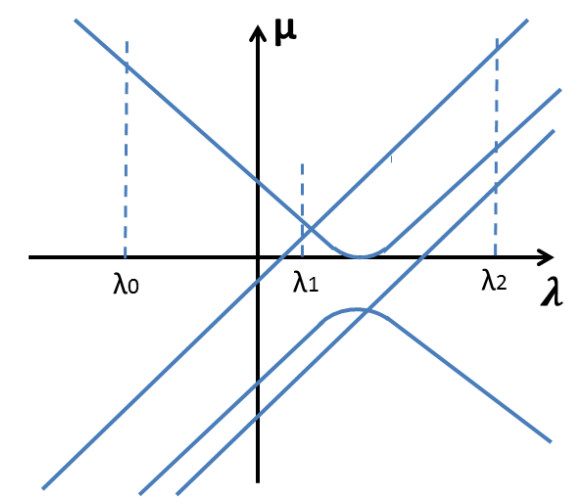

Then the index sequence of a quadric pencil can be defined as follows. Let $\lambda_{1}<\cdots<\lambda_{r}$ be all the distinct real roots of $f(\lambda)=0$, and let $q_{1}<\cdots<q_{r-1}$ be arbitrary real numbers separating the roots $\lambda_{i}$, i.e.,

$$
-\infty<\lambda_{1}<q_{1}<\lambda_{2}<\cdots<q_{r-1}<\lambda_{r}<+\infty .
$$

Denote by $s_{i}=\operatorname{Id}\left(q_{i}\right), i=1,2, \cdots, r-1$, and $s_{0}=\operatorname{Id}(-\infty)$, $s_{r}=\operatorname{Id}(+\infty)$. The index sequence of the quadric pencil $\lambda A-B$ is defined as

$$
\left\langle s_{0}\left|s_{1}\right| \cdots\left|s_{r-1}\right| s_{r}\right\rangle
$$

where $\mid$ stands for a real root of $f(\lambda)=0$. For example, the index sequences of the three quadric pencils formed by the two ellipsoids in Fig. 8 are $\langle 1|0| 1|2| 3\rangle,\langle 1|2| 1|2| 3\rangle,\langle 1|2| 3|2| 3\rangle$, respectively. When the characteristic polynomial has multiple roots, we replace $\mid$ by 2 of $p$ times in a row to denote a $p \times p$ Jordan block and use $\imath \cdots \imath_{+}$or $\imath \cdots \imath_{-}$to denote the sign of the block; for example, $\imath_{+} \mid$means a $2 \times 2$ block of sign + and a $1 \times 1$ block associated to the same real root. A change in $\operatorname{Id}(\lambda)$ indicates a real root of the characteristic polynomial. The value of $\Delta_{i}:=s_{i}-s_{i-1}$ depends on the Jordan blocks associated to the root $\lambda_{i}, i=1, \cdots, r: \Delta_{i}= \pm 1$ across a $1 \times 1$ or a $3 \times 3$ block, and $\Delta_{i}=0$ across a $2 \times 2$ block; hence $\Delta_{i}= \pm 1$ if the Jordan block associated to $\lambda_{i}$ is $\imath_{ \pm} \mid$. Please refer to the appendix or [Tu et al. 2009] for more details. Tu et al. showed that index sequences characterize the intersection morphology of the intersection curves of two quadrics.

LEMMA 4.2. [Tu et al. 2009] If two different index sequences are equivalent under the following rules, the intersection curves of the two pairs of quadrics have the same morphology.

(1) Rotation rule:

$$
\begin{aligned}
& \left\langle s_{0}\left|s_{1}\right| \cdots \mid s_{r}\right\rangle \sim\left\langle 4-s_{r}\left|s_{0}\right| \cdots\left|s_{r-2}\right| s_{r-1}\right\rangle, \\
& \left\langle s_{0}\left|s_{1}\right| \cdots \mid s_{r}\right\rangle \sim\left\langle s_{1}\left|s_{2}\right| \cdots\left|s_{r}\right| 4-s_{1}\right\rangle .
\end{aligned}
$$

(2) Reversal rule:

(3) Complement rule:

$$
\left\langle s_{0}\left|s_{1}\right| \cdots \mid s_{r}\right\rangle \sim\left\langle s_{r}\left|s_{r-1}\right| \cdots\left|s_{1}\right| s_{0}\right\rangle .
$$

$$
\left\langle s_{0}\left|s_{1}\right| \cdots \mid s_{r}\right\rangle \sim\left\langle 4-s_{0}\left|4-s_{1}\right| \cdots \mid 4-s_{r}\right\rangle .
$$

For example, in Fig. 8(b) and (c), the intersection curves of the two pairs of ellipsoids have the same morphology which contains two real conics, and the corresponding index sequences $\langle 1|2| 3|2| 3\rangle$ and $\langle 1|2| 1|2| 3\rangle$ are equivalent under the rotation rule; however, Fig. 8(a) gives a different intersection morphology because $\langle 1|0| 1|2| 3\rangle$ is not equivalent to the above two index sequences under the three rules.

Nevertheless, index sequences are still not enough to characterize arrangements. A simple example is that Fig. 8(b) and (c) have non-equivalent arrangements but equivalent index sequences. Therefore, further algebraic considerations have to be taken into account to characterize arrangements of a pair of ellipsoids.

\subsection{Modified index sequence}

The geometric interpretation of Lemma 4.2 is that taking any two representatives from a quadric pencil generated by $\mathcal{A}$ and $\mathcal{B}$ will give exactly the same intersection curve as that of $\mathcal{A}$ and $\mathcal{B}$. However, the arbitrary choice of two ellipsoids from the pencil may create a different arrangement. A simple example is that exchanging the two ellipsoids $\mathcal{A}$ and $\mathcal{B}$ with each other usually gives a totally different arrangement. In fact, the rotation rule and reversal rule can no longer characterize equivalent arrangements.

Therefore, the two ellipsoids $\mathcal{A}$ and $\mathcal{B}$ need to be specified in the index sequence. Note that in the pencil $\lambda A-B, \lambda=0$ gives $\mathcal{B}$ and $\lambda= \pm \infty$ gives $\mathcal{A}$. Also note that in this paper both $\mathcal{A}$ and $\mathcal{B}$ are ellipsoids, hence $\operatorname{Id}(+\infty)=\operatorname{Index}(A)=3$ and $\operatorname{Id}(-\infty)=\operatorname{rank}(A)-\operatorname{Id}(+\infty)=1$. So the specification of $\mathcal{A}$ and $\mathcal{B}$ can be achieved by fixing $\operatorname{Id}(+\infty)=3, \operatorname{Id}(-\infty)=$ 1 , and labeling the interval containing $\lambda=0$ in the index sequence by symbol ' $\wedge$ ' as

$$
\left\langle 1\left|\operatorname{Id}\left(q_{1}\right)\right| \cdots|\widehat{\operatorname{Id}(0)}| \cdots\left|\operatorname{Id}\left(q_{r-1}\right)\right| 3\right\rangle
$$

where $q_{1}<\ldots<q_{r-1}$ are real numbers separating the real roots of $f(\lambda)=0$. Note that $\widehat{\operatorname{Id}(0)}=1$. We call this sequence the modified index sequence of ellipsoids $\mathcal{A}$ and $\mathcal{B}$. Note that $\lambda=0$ can not be a root of $f(\lambda)=0$ since $\operatorname{det}(B) \neq 0$, hence the modified index sequence is well defined. This specification of ellipsoids $\mathcal{A}$ and $\mathcal{B}$ yields the following result.

Proposition 4.3. If two pairs of ellipsoids have the same modified index sequences, then these two pairs of the ellipsoids have equivalent arrangements.

As stated before, $\langle 1|2| 3|2| 3\rangle$ and $\langle 1|2| 1|2| 3\rangle$ are equivalent under the rotation rule; however, their corresponding modified index sequences $\langle\hat{1}|2| 3|2| 3\rangle$ and $\langle\hat{1}|2| 1|2| 3\rangle$ tell the difference between two arrangements.

\section{OUTLINE OF ENUMERATION}

We enumerate all non-equivalent arrangements of a pair of ellipsoids in the following outline.

(1) Select all non-equivalent intersection morphologies of the pencil formed by two ellipsoids; 
(2) Enumerate all modified index sequences for each intersection morphology in step (1) and derive the corresponding arrangement for each modified index sequence of two ellipsoids;

(3) Identify those equivalent arrangements generated from non-equivalent intersection morphologies listed in step (2) to give the final classification of arrangements of two ellipsoids.

We provide details for each of the above steps in the following subsections.

\subsection{Enumeration of Intersection Morphologies}

$\mathrm{Tu}$ et al. provided a full enumeration of the intersection morphologyies of two quadrics in $\mathbb{P R}^{3}$ [Tu et al. 2009]. Since we are focusing on a pair of ellipsoids whose intersection is a closed curve in $\mathbb{R}^{3}$, we first extract all possible intersection morphologies of a pair of ellipsoids from the full list in [Tu et al. 2009 ] by excluding those unbounded ones. For example, the intersection morphology containing a real line or a real space cubic curve in [ Tu et al. 2009] is not a possible intersection of two ellipsoids. The first columns in Table 1(a)-(c) are all non-equivalent intersection morphologies for a pair of ellipsoids, where solid lines/curves are the real components and the dashed ones are the imaginary components, and thick lines/curves have multiplicity two.

\subsection{Enumeration of Arrangements}

For the quadric pencil formed by a pair of ellipsoids $\mathcal{A}$ and $\mathcal{B}$, different choices of two quadrics from the pencil give the same intersection morphology, but may provide non-equivalent arrangements. For example, suppose that the intersection of $\mathcal{A}$ and $\mathcal{B}$ is empty in $\mathbb{R}^{3}$, then there can be three non-equivalent arrangements: 1$) \mathcal{A}$ and $\mathcal{B}$ are separate; 2) $\mathcal{A}$ contains $\mathcal{B}$; and 3) $\mathcal{A}$ is contained in $\mathcal{B}$. Hence we next enumerate all possible non-equivalent arrangements for each enumerated intersection morphology for a pair of ellipsoids. We proceed as follows.

(1) Enumerate all possible modified index sequences for each intersection morphology;

(2) Analyze the arrangement for each enumerated modified sequence.

Each intersection morphology has an index sequence corresponding to it, as is listed in Table 1-3 in [Tu et al. 2009]. For each of these index sequences, we first enumerate all its equivalent ones under the rotation rule, reversal rule and complement rule, and then select those of the form $\langle 1|.| .|| 3$. (| can be replaced by notions for Jordan blocks of size other than one), and finally label the interval containing $\lambda=0$.

For example, the first case of intersection morphology in Table 1 of [Tu et al. 2009] is two separated real loops, whose corresponding index sequence equivalence is $\langle 1|2| 1|2| 3\rangle$. Using the three rules we get another seven of its equivalent index sequences: $\langle 2|1| 2|1| 2\rangle,\langle 3|2| 1|2| 1\rangle,\langle 2|3| 2|1| 2\rangle,\langle 3|2| 3|2| 1\rangle$, $\langle 2|3| 2|3| 2\rangle$,

$\langle 1|2| 3|2| 3\rangle,\langle 2|1| 2|3| 2\rangle$, among which only $\langle 1|2| 1|2| 3\rangle$ and

$\langle 1|2| 3|2| 3\rangle$ are possible for two ellipsoids. Taking the interval containing $\lambda=0$ into account, all possible modified index sequences for this intersection morphology are $\langle\hat{1}|2| 1|2| 3\rangle$, $\langle 1|2| \hat{1}|2| 3\rangle,\langle\hat{1}|2| 3|2| 3\rangle$.

Next we show how to analyze the arrangement for a specific modified index sequence. We start from the simplest case that the characteristic polynomial has four simple real roots by enumerating all modified index sequences under this situation.

TheOREM 5.1. Given a pair of ellipsoids $\mathcal{A}$ and $\mathcal{B}$, if the characteristic polynomial has four different real roots, the quadric pencil has the following possible modified index sequences and corresponding arrangements:

(1) $\langle 1|0| \hat{1}|2| 3\rangle-\mathcal{A}$ and $\mathcal{B}$ are separate (case 1 );

(2) $\langle\hat{1}|0| 1|2| 3\rangle-\mathcal{A}$ contains $\mathcal{B}$ (case 2$) ;$

(3) $\langle\hat{1}|2| 3|4| 3\rangle-\mathcal{B}$ contains $\mathcal{A}$ (case 3);

(4) $\langle\hat{1}|2| 1|2| 3\rangle-\mathcal{B}$ penetrates $\mathcal{A}$, and has two components exterior to $\mathcal{A}$ (case 4 );

(5) $\langle\hat{1}|2| 3|2| 3\rangle-\mathcal{A}$ penetrates $\mathcal{B}$, and has two components exterior to $\mathcal{B}$ (case 5 );

Proof. From Table 1 in [Tu et al. 2009], when the characteristic polynomial has four different real roots, there are two index sequences $\langle 1|2| 1|2| 3\rangle$ and $\langle 0|1| 2|3| 4\rangle$ corresponding to it. In the above we have shown that all possible modified index sequences for $\langle 1|2| 1|2| 3\rangle$ are $\langle\hat{1}|2| 1|2| 3\rangle,\langle 1|2| \hat{1}|2| 3\rangle,\langle\hat{1}|2| 3|2| 3\rangle$. Using a similar treatment we know that all possible modified index sequences for $\langle 0|1| 2|3| 4\rangle$ are $\langle 1|0| \hat{1}|2| 3\rangle,\langle\hat{1}|0| 1|2| 3\rangle$ and $\langle\hat{1}|2| 3|4| 3\rangle$.

Now let $\lambda_{1}<\lambda_{2}<\lambda_{3}<\lambda_{4}$ be the four distinct real roots of the characteristic function. By Lemma 1.4 in the appendix, the matrices $A$ and $B$ are simultaneously congruent to the following canonical forms:

$A_{0}=\operatorname{diag}\left(\varepsilon_{1}, \varepsilon_{2}, \varepsilon_{3}, \varepsilon_{4}\right)$, and $B_{0}=\operatorname{diag}\left(\varepsilon_{1} \lambda_{1}, \varepsilon_{2} \lambda_{2}, \varepsilon_{3} \lambda_{3}, \varepsilon_{4} \lambda_{4}\right)$,

where $\varepsilon_{i}=+1$ if $s_{i}-s_{i-1}=1 ; \varepsilon_{i}=-1$ if $s_{i-1}-s_{i}=1$. Note that $f(\lambda)$ is, up to the determinant of $A$, the characteristic polynomial of $A^{-1} B$.

$0)$. The modified index sequence $\langle 1|2| \hat{1}|2| 3\rangle$ is invalid. To see this, we show that $\operatorname{Id}\left(\lambda^{*}\right) \neq 2$ for $\lambda^{*} \in\left(\lambda_{1}, \lambda_{2}\right)$. Since $A$ and $B$ are simultaneously congruent to $A_{0}$ and $B_{0}$, we have $\lambda^{*} A_{0}-B_{0}=\operatorname{diag}\left(-\lambda^{*}+\lambda_{1}, \lambda^{*}-\lambda_{2}, \lambda^{*}-\lambda_{3}, \lambda^{*}-\lambda_{4}\right)$, which are of signs $(-,-,-,-)$. So $\operatorname{Id}\left(\lambda^{*}\right)=0$.

$1)$. If the modified index sequence is $\langle 1|0| \hat{1}|2| 3\rangle, f(\lambda)=0$ has two negative simple roots. By Lemma $4.1, \mathcal{A}$ and $\mathcal{B}$ are separate.

$2)$. If the modified index sequence is $\langle\hat{1}|0| 1|2| 3\rangle$, then in the canonical forms $\varepsilon_{1}=-1, \varepsilon_{2}=\varepsilon_{3}=\varepsilon_{4}=1$, i.e.

$$
A_{0}=\operatorname{diag}(-1,1,1,1) \text {, and } B_{0}=\operatorname{diag}\left(-\lambda_{1}, \lambda_{2}, \lambda_{3}, \lambda_{4}\right) .
$$

Letting $x=0$ be the plane at infinity gives the affine realization of the quadric pair in $\mathbb{R}^{3}: \mathcal{A}_{0}$ is a unit sphere, and $\mathcal{B}_{0}$ is an ellipsoid with equation $\frac{\lambda_{2}}{\lambda_{1}} y^{2}+\frac{\lambda_{3}}{\lambda_{1}} z^{2}+\frac{\lambda_{4}}{\lambda_{1}} w^{2}=1$. Noticing that $\lambda_{1}<\lambda_{2}<\lambda_{3}<\lambda_{4}$, the three axes of ellipsoid $\mathcal{B}_{0}$ are all smaller than one. Clearly, the sphere $\mathcal{A}_{0}$ contains the ellipsoid $\mathcal{B}_{0}$. 
3). If the modified index sequence is $\langle\hat{1}|2| 3|4| 3\rangle$, then $\varepsilon_{1}=$ $\varepsilon_{2}=\varepsilon_{3}=1, \varepsilon_{4}=-1$. So

$$
A_{0}=\operatorname{diag}(1,1,1,-1) \text {, and } B_{0}=\operatorname{diag}\left(\lambda_{1}, \lambda_{2}, \lambda_{3},-\lambda_{4}\right) .
$$

Letting $w=0$ be the plane at infinity gives the affine realization of the quadric pair in $\mathbb{R}^{3}: \mathcal{A}_{0}$ is a unit sphere, and $\mathcal{B}_{0}$ is an ellipsoid with equation $\frac{\lambda_{1}}{\lambda_{4}} x^{2}+\frac{\lambda_{2}}{\lambda_{4}} y^{2}+\frac{\lambda_{3}}{\lambda_{4}} z^{2}=1$. Since $\lambda_{1}<\lambda_{2}<\lambda_{3}<\lambda_{4}$, the three axes of the ellipsoid $\mathcal{B}_{0}$ are all bigger than one. Clearly the ellipsoid $\mathcal{B}_{0}$ contains the sphere $\mathcal{A}_{0}$.

4). If the modified index sequence is $\langle\hat{1}|2| 1|2| 3\rangle$, then $\varepsilon_{1}=$ $\varepsilon_{3}=\varepsilon_{4}=1, \varepsilon_{2}=-1$. The two canonical quadrics are

$$
A_{0}=\operatorname{diag}(1,-1,1,1) \text {, and } B_{0}=\operatorname{diag}\left(\lambda_{1},-\lambda_{2}, \lambda_{3}, \lambda_{4}\right) .
$$

Letting $y=0$ be the plane at infinity gives the affine realization of the quadric pair in $\mathbb{R}^{3}: \mathcal{A}_{0}$ is a unit sphere, and $\mathcal{B}_{0}$ is an ellipsoid with equation $\frac{\lambda_{1}}{\lambda_{2}} x^{2}+\frac{\lambda_{3}}{\lambda_{2}} z^{2}+\frac{\lambda_{4}}{\lambda_{2}} w^{2}=1$ Since $0<\lambda_{1}<\lambda_{2}<\lambda_{3}<\lambda_{4}$, the two axes of the ellipsoid $\mathcal{B}_{0}$ parallel to $z$-axis and $w$-axis are bigger than one, and the axis parallel to $y$-axis is smaller than one. Clearly the ellipsoid $\mathcal{B}_{0}$ penetrates the sphere $\mathcal{A}_{0}$ in $x$-direction.

$5)$. If the modified index sequence is $\langle\hat{1}|2| 3|2| 3\rangle$, then $\varepsilon_{1}=$ $\varepsilon_{2}=\varepsilon_{4}=1, \varepsilon_{3}=-1$. The two canonical quadrics are

$$
A_{0}=\operatorname{diag}(1,1,-1,1) \text {, and } B_{0}=\operatorname{diag}\left(\lambda_{1}, \lambda_{2},-\lambda_{3}, \lambda_{4}\right) .
$$

Letting $z=0$ be the plane at infinity gives the affine realization of the quadric pair in $\mathbb{R}^{3}: \mathcal{A}_{0}$ is a unit sphere, and $\mathcal{B}_{0}$ is an ellipsoid with equation $\frac{\lambda_{1}}{\lambda_{3}} x^{2}+\frac{\lambda_{2}}{\lambda_{3}} y^{2}+\frac{\lambda_{4}}{\lambda_{3}} w^{2}=1$. Since $0<\lambda_{1}<\lambda_{2}<\lambda_{3}<\lambda_{4}$, the two axes of the ellipsoid $\mathcal{B}_{0}$ parallel to $x$-axis and $y$-axis are bigger than one, and the axis parallel to $w$-axis is smaller than one. Clearly the ellipsoid $\mathcal{A}_{0}$ penetrates the sphere $\mathcal{B}_{0}$ in $x$-direction.

Since there are many intersection morphologies enumerated for a pair of ellipsoids, we organize the detailed proofs on the arrangements similar to Theorem 5.1 for other modified index sequences in Section 3 of the appendix. The final enumeration results are shown in Table 1(a)-(c), where the first columns list all possible intersection morphologies for a pair of ellipsoids, and the second columns and the third column$\mathrm{s}$ are the modified index sequences and the illustrations of corresponding arrangements.

\subsection{Classifications of equivalent arrangements}

Two arrangements associated to different intersection morphologies of two ellipsoids can be equivalent to each other. For example, case 1 in Table 1(a) and case 21 in Table 1(b), though associated to different intersection morphologies, the two arrangements are equivalent. Therefore, we need to combine equivalent arrangements and choose only a representative modified index sequence for each equivalent class. Table 2 is our final classification result for 21 non-equivalent arrangements, where the second column are the case numbers from Table 1(a)-(c) that have equivalent arrangements, and the third column are the representative modified sequences. Note that we choose the representative modified sequence in each class as the one with the least degenerate degree, i.e, the one with less multiple roots in the characteristic polynomial. More details on the degenerate degrees of modified index sequences will be given shortly in Section 7 .

Table 1(a): Enumeration list I of arrangements of a pair of ellipsoids by modified index sequences.

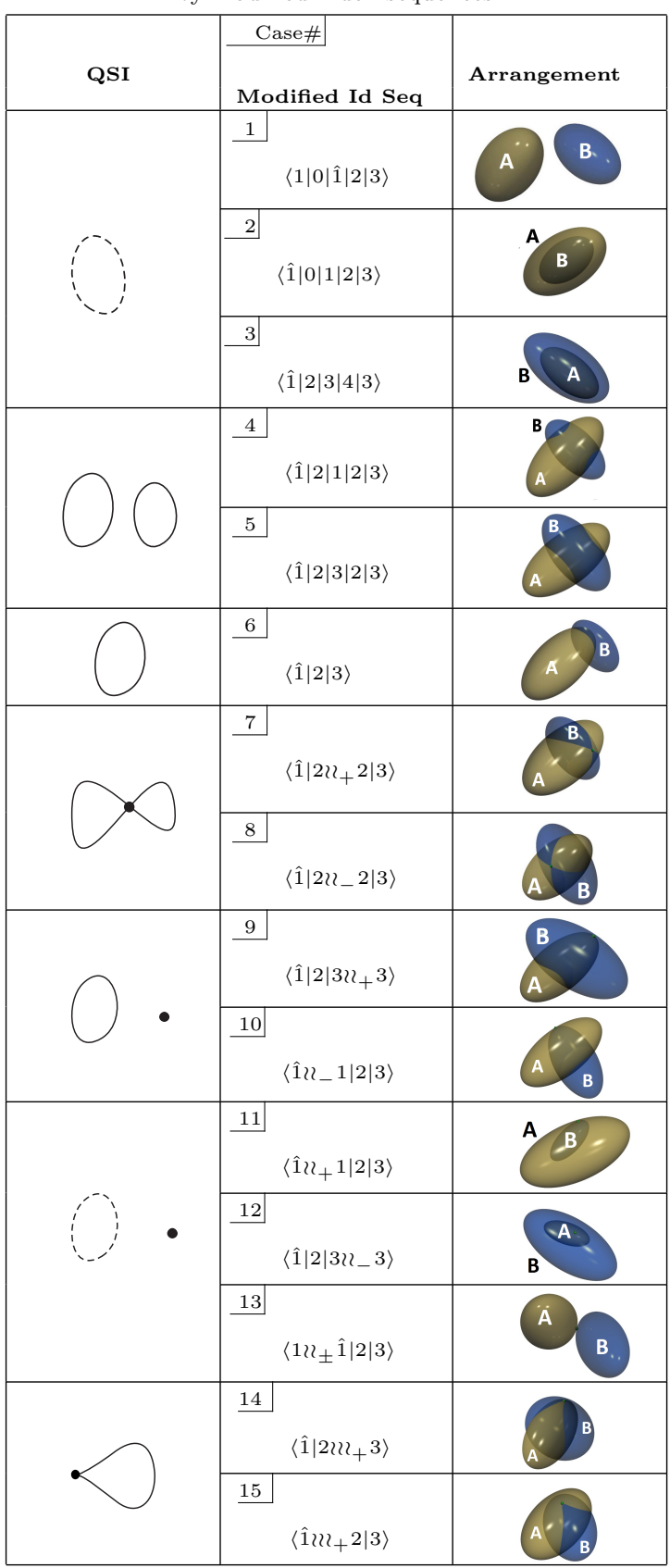

\section{DETERMINATION OF ARRANGEMENTS}

We provide Algorithm 1 for determination of the arrangement of two given ellipsoids. The most costly steps of the algorithm 
Table 1(b): Enumeration list II of arrangements of a pair of ellipsoids by modified index sequences.

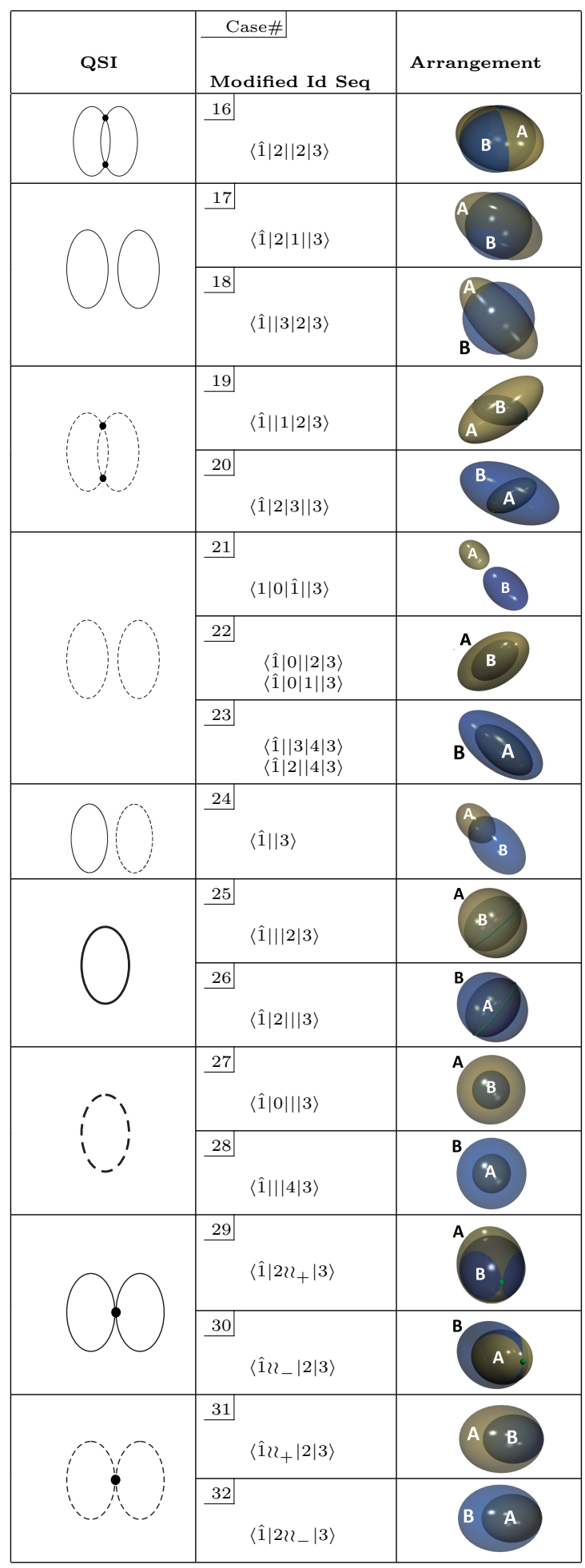

Table 1(c): Enumeration list III of arrangements of a pair of ellipsoids by modified index sequences.

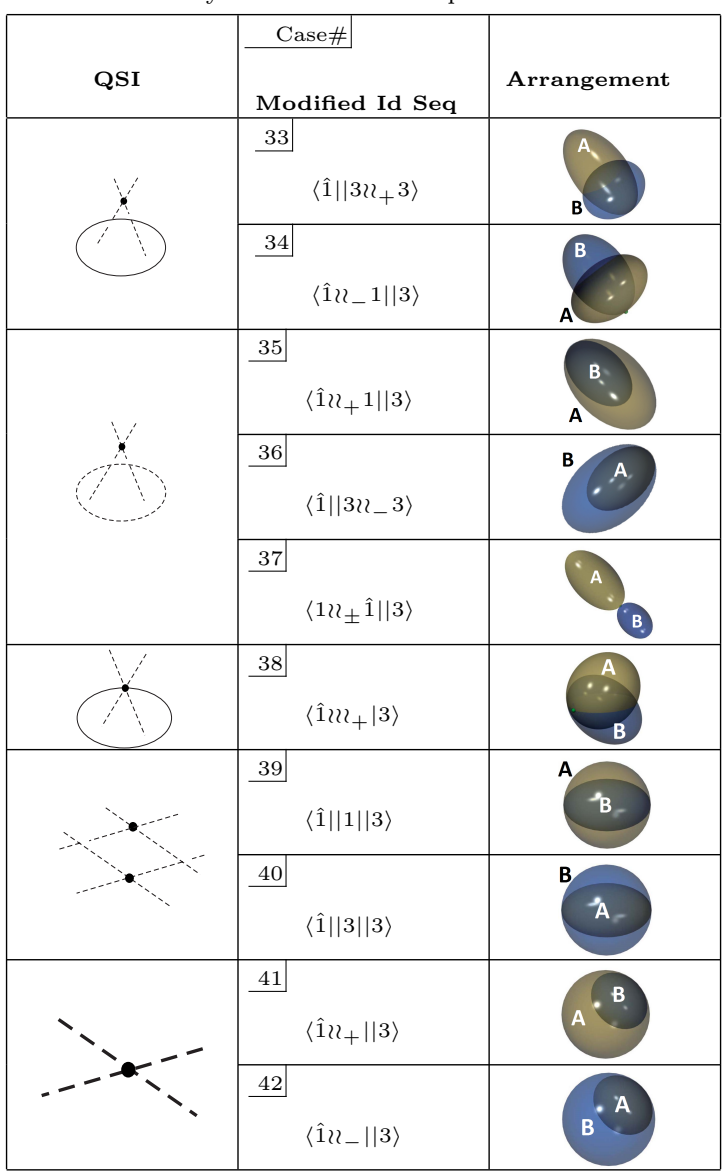

are, in increasing order, steps 3, 4, 5, 6 and 7 , which require the separation of real roots of a polynomial of degree $\leq 4$, to compute their multiplicities, to determine the number of positive roots and the sign of a polynomial of degree $\leq 4$ at the real roots of another polynomial of degree $\leq 4$. These operations can be done efficiently, in $O(1)$ arithmetic operations, using static Sturm sequences based on the approach of [Emiris and Tsigaridas 2008] for polynomials of degree $\leq 4$. Readers are referred to [Tu et al. 2009] for further explanations on the computation of signature sequences. The main difference of this algorithm with that of [Tu et al. 2009] is that we need to label the interval containing $\lambda=0$ in the modified index sequence (step 5). This is computed in $O(1)$ arithmetic operations from the sign changes of the Sturm sequence at 0 and at $\infty$.

Note that in step 7 , the signature $\left(p_{i}, n_{i}\right)$ at a root $\lambda_{i}$ are the sign variations of $\left\{c_{0}\left(\lambda_{i}\right), c_{1}\left(\lambda_{i}\right), c_{2}\left(\lambda_{i}\right), c_{3}\left(\lambda_{i}\right), 1\right\}$ and $\left\{c_{0}\left(\lambda_{i}\right),-c_{1}\left(\lambda_{i}\right), c_{2}\left(\lambda_{i}\right),-c_{3}\left(\lambda_{i}\right), 1\right\}$. Since the root $\lambda_{i}$ is generically not rational hence represented by an isolated interval $\left[a_{i}, b_{i}\right]$, the signs of $c_{j}\left(\lambda_{i}\right), j=1,2,3$ can be computed by $V_{c_{0}, c_{j}}\left(a_{i}\right)-V_{c_{0}, c_{j}}\left(b_{i}\right)$, where $V_{c_{0}, c_{j}}$ means the number of sign variations of the Sturm sequence of $c_{0}(\lambda)$ and $c_{0}^{\prime} c_{j}(\lambda)$. Then 
Table 2: A total number of 21 equivalent classes of all the enumerated arrangements.

\begin{tabular}{|c|c|c|c|}
\hline AR \# & Index seq \# & Rep. Id seq. & Arrangement \\
\hline$A R_{1}$ & $\{1,21\}$ & $\langle 1|0| \hat{1}|2| 3\rangle$ & \\
\hline$A R_{2}$ & $\{2,22,27\}$ & $\langle\hat{1}|0| 1|2| 3\rangle$ & \\
\hline$A R_{3}$ & $\{3,23,28\}$ & $\langle\hat{1}|2| 3|4| 3\rangle$ & \\
\hline$A R_{4}$ & $\{4,17\}$ & $\langle\hat{1}|2| 1|2| 3\rangle$ & \\
\hline$A R_{5}$ & $\{5,18\}$ & $\langle\hat{1}|2| 3|2| 3\rangle$ & \\
\hline$A R_{6}$ & $\{6,24\}$ & $\langle\hat{1}|2| 3\rangle$ & \\
\hline$A R_{7}$ & $\{7,29\}$ & $\langle\hat{1}|2 \imath \imath+2| 3\rangle$ & \\
\hline$A R_{8}$ & $\{8,30\}$ & $\left\langle\hat{1}\left|2 \imath \imath_{-} 2\right| 3\right\rangle$ & \\
\hline$A R_{9}$ & $\{9,33\}$ & $\left\langle\hat{1}|2| 3 \imath_{+} 3\right\rangle$ & \\
\hline$A R_{10}$ & $\{10,34\}$ & $\langle\hat{\imath} \varkappa-1|2| 3\rangle$ & \\
\hline$A R_{11}$ & $\{11,31,35,41\}$ & $\left\langle\hat{1} \imath_{+}+1|2| 3\right\rangle$ & \\
\hline$A R_{12}$ & $\{12,32,36,42\}$ & $\left\langle\hat{1}|2| 3 \imath_{-} 3\right\rangle$ & \\
\hline$A R_{13}$ & $\{13,37\}$ & $\langle 12 \imath+\hat{1}|2| 3\rangle$ & \\
\hline$A R_{14}$ & $\{14\}$ & $\left\langle\hat{1} \mid 2 u_{+}+3\right\rangle$ & \\
\hline$A R_{15}$ & $\{15\}$ & $\langle\hat{1} \geq 2+2 \mid 3\rangle$ & \\
\hline$A R_{16}$ & $\{16\}$ & $\langle\hat{1}|2||2| 3\rangle$ & \\
\hline$A R_{17}$ & $\{19,39\}$ & $\langle\hat{1}|| 1|2| 3\rangle$ & \\
\hline$A R_{18}$ & $\{20,40\}$ & $\langle\hat{1}|2| 3|| 3\rangle$ & \\
\hline$A R_{19}$ & $\{25\}$ & $\langle\hat{1}|||2| 3\rangle$ & \\
\hline$A R_{20}$ & $\{26\}$ & $\langle\hat{1}|2||| 3\rangle$ & \\
\hline$A R_{21}$ & $\{38\}$ & $\left\langle\hat{1} 22 u_{+} \mid 3\right\rangle$ & \\
\hline
\end{tabular}

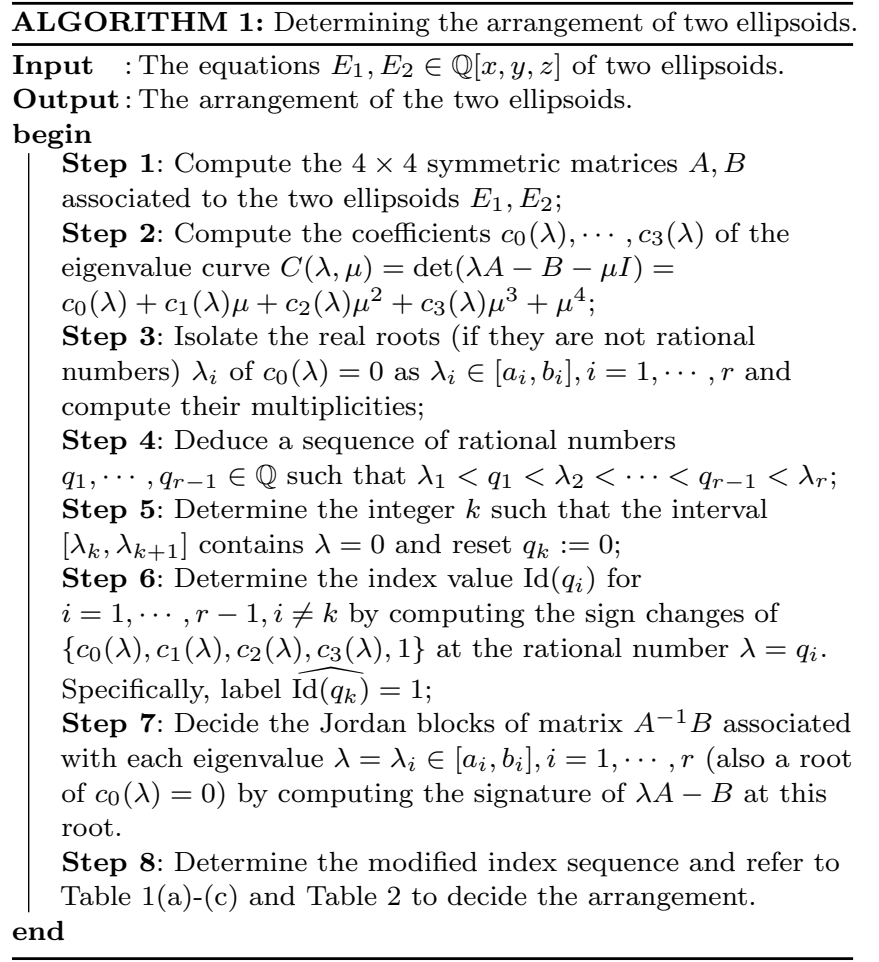

see how to decide the Jordan blocks of $A^{-1} B$ associated with its eigenvalue $\lambda_{i}$ (which is also a root of the characteristic polynomial $f(\lambda)$ ) from the signature in section 2 of the appendix. Related materials can refer to page 325 of [Tu et al. 2009].

Example 6.1. Consider two ellipsoids $\mathcal{A}: x^{2}+y^{2}+z^{2}+2 x=$ 0 and $\mathcal{B}: \frac{x^{2}}{4}+4 y^{2}+4 z^{2}+x=0$. The eigenvalue curve $\mathcal{C}$ (shown in Fig. 10) has the equation

$$
\begin{aligned}
& C(\lambda, \mu)=\mu^{4}+\left(\frac{33}{4}-3 \lambda\right) \mu^{3}+\left(2 \lambda^{2}-\frac{31}{2} \lambda+\frac{71}{4}\right) \mu^{2}+\left(\lambda^{3}\right. \\
& \left.-\frac{7}{4} \lambda^{2}-\frac{19}{2} \lambda+2\right) \mu-\lambda^{4}+9 \lambda^{3}-\frac{97}{4} \lambda^{2}+18 \lambda-4=0 .
\end{aligned}
$$

The characteristic polynomial

$$
c_{0}(\lambda)=-\lambda^{4}+9 \lambda^{3}-\frac{97}{4} \lambda^{2}+18 \lambda-4
$$

has two double roots $\lambda_{1}=\frac{1}{2}, \lambda_{2}=4$. Choose $q_{1}=1 \in$ $\left[\lambda_{1}, \lambda_{2}\right]$. The sign change of $\left\{c_{0}(\lambda), c_{1}(\lambda), c_{2}(\lambda), c_{3}(\lambda), 1\right\}$ at $q_{1}$ is 1 , hence $\operatorname{Id}\left(q_{1}\right)=1$. Since $\operatorname{Id}(-\infty)=1, \operatorname{Id}(+\infty)=$ 3 , considering the index variation rule and that $\lambda=0 \in$ $\left(-\infty, \lambda_{1}\right]$, the modified index sequence has the form $\langle\hat{1} \imath 21|| 3\rangle$. Computing the sign variations of $\left\{c_{0}(\lambda), c_{1}(\lambda), c_{2}(\lambda), c_{3}(\lambda), 1\right\}$ and $\left\{c_{0}(\lambda),-c_{1}(\lambda), c_{2}(\lambda),-c_{3}(\lambda), 1\right\}$ at $\lambda=\lambda_{1}$, the signature at $\lambda_{1}$ is $(1,2)$, which by Table 1 in the appendix suggests that the Jordan block of size two has negative sign. Therefore, the modified sequence is $\left\langle\hat{1} \chi_{-} 1 \| 3\right\rangle$. This modified sequence corresponds to the arrangement of case 34 in Table 1(c), which belongs to the 10th equivalent class of arrangements in Table 2 . 


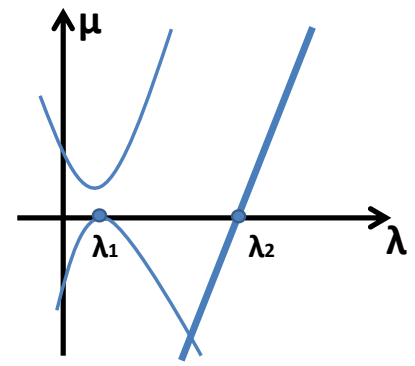

Fig. 10. The eigenvalue curve of Example 6.1, where the thick line is a double line.

\section{STRATIFICATION OF ALL ARRANGEMENTS}

Table 2 shows the final 21 equivalent classes of all the enumerated arrangements. We next aim at building a connection diagram that indicates how these 21 arrangements transform to each other under continuous transformations or deformations.

Definition 7.1. Two arrangements $\mathcal{A R}_{1}$ and $\mathcal{A R}_{2}$ are connected, denoted by $\mathcal{A R}_{1} \sim \mathcal{A} \mathcal{R}_{2}$, if there is a moving ellipsoid pair $\mathcal{A}(t), \mathcal{B}(t)$ such that

$$
\mathcal{A R}(\mathcal{A}(t), \mathcal{B}(t))=\mathcal{A} \mathcal{R}_{1} \text { for } t_{1} \leq t<t_{2}
$$

and

$$
\mathcal{A R}\left(\mathcal{A}\left(t_{2}\right), \mathcal{B}\left(t_{2}\right)\right)=\mathcal{A} \mathcal{R}_{2},
$$

which means that the arrangement of the moving ellipsoid changes from $\mathcal{A R}_{1}$ to $\mathcal{A R}_{2}$ without going through a third arrangements during time span $\left[t_{1}, t_{2}\right]$; otherwise the arrangements are said to be disconnected. Two modified index sequences are connected (disconnected) if their corresponding arrangements are connected (disconnected).

For each equivalent class of arrangements shown in Table 2 , we pick the representative modified index sequence as the one with the least multiplicity in real roots. For example, Case 1 and Case 21 are equivalent, with index sequences $\langle 1|0| \hat{1}|2| 3\rangle$ and $\langle 1|0| \hat{1}|| 3\rangle$, respectively. We choose $\langle 1|0| \hat{1}|2| 3\rangle$ as the representative, as shown in the third column. Then we classify all these 21 representative modified index sequences by the multiplicities and numbers of singularities on the intersection curve of the two ellipsoids as follows.

(1) No singularity on the intersection curve: $\langle 1|0| \hat{1}|2| 3\rangle,\langle\hat{1}|0| 1|2| 3\rangle,\langle\hat{1}|2| 3|4| 3\rangle,\langle\hat{1}|2| 1|2| 3\rangle,\langle\hat{1}|2| 3|2| 3\rangle$, $\langle\hat{1}|2| 3\rangle$.

(2) One double singularity on the intersection curve:

$\left\langle\hat{1}\left|2 \imath_{+} 2\right| 3\right\rangle,\left\langle\hat{1}\left|2 \imath_{-} 2\right| 3\right\rangle,\left\langle\hat{1}|2| 3 \imath_{+} 3\right\rangle,\left\langle\hat{1} \imath_{-} 1|2| 3\right\rangle$, $\left\langle\hat{1} \imath_{+} 1|2| 3\right\rangle,\left\langle\hat{1}|2| 3 \imath_{-} 3\right\rangle,\left\langle 1 \imath_{+} \hat{1}|2| 3\right\rangle$.

(3) One triple singularity or two double singularities on the intersection curve:

$\left\langle\hat{1} \mid 2 \imath_{+} 3\right\rangle,\left\langle\hat{1} \varkappa_{+} 2 \mid 3\right\rangle$ (one triple singularity);

$\langle\hat{1}|2||2| 3\rangle,\langle\hat{1}|| 1|2| 3\rangle,\langle\hat{1}|2| 3|| 3\rangle$ (two double singularities).

(4) The whole intersection curve is singular:

$\langle\hat{1}|2||| 3\rangle,\langle\hat{1}|||2| 3\rangle,\left\langle\hat{1} 2 \chi_{+} \mid 3\right\rangle$.
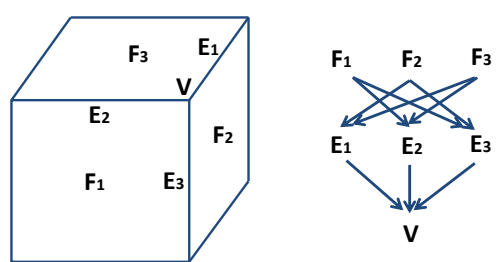

Fig. 11. A face-edge-vertex structure. $F_{i}$ are faces, $E_{i}$ are edges and $V$ is a vertex. $F_{3} \sim E_{1}$ and $E_{1} \sim V$, hence $F_{3} \sim V$, where $\sim$ means the connection between elements of neighboring dimensions. Other connections are similarly derived.

Suppose that $A R_{i}, A R_{j}, A R_{k}, A R_{l}$ are four different arrangements from the above (1)-(4) degenerate categories. If $A R_{i} \sim A R_{j}, A R_{j} \sim A R_{k}$ and $A R_{k} \sim A R_{l}$, then we shall have $A R_{i} \sim A R_{k}, A R_{j} \sim A R_{l}$ and also $A R_{i} \sim A R_{l}$. This is analogue to the boundary structure of a volume in Figure 11, where the faces, edges and vertices have neighboring dimensions 2,1,0. The face $F_{3}$ connects with the edges $E_{1}$ and $E_{2}$, and the edges $E_{1}$ and $E_{2}$ connect with the vertex $V$, then we naturally have that face $F_{3}$ connects with the vertex $V$. Therefore, to explore a compete connection diagram of all these 21 arrangements, we need only to investigate the connections between the arrangements from neighboring cases, i.e, (1) and (2), (2) and (3), (3) and (4). Fig. 12, 13, 14 show the connection diagrams of arrangements for neighboring cases, where the upper-left label of each arrangement is from the arrangement numbers $1-21$ shown as $A R_{1}$ to $A R_{21}$ in Table 2. These three diagrams together provide a full stratification of the 21 non-equivalent arrangements. We show the full connection diagram in Table 3 . Note that in Table 3, if $A R_{i} \sim A R_{j}$ and $A R_{j} \sim A R_{k}$, where $A R_{i}, A R_{j}, A R_{k}$ are with higher multiplicity or more singularities in sequence, then $A R_{i} \sim A R_{k}$ is also marked as a connection of arrangements. Due to space limit, we cannot include the proofs for all the cases in Table 3 here so refer the reader to the appendix in the online supplementary materials. However, because all these cases share a common key idea but with necessary variations to accommodate their respective special aspects, to help the reader appreciate the key idea, we give below only the proof for the connection of $A R_{5} \sim A R_{9}$.

TheOrEm 7.2. $\langle\hat{1}|2| 3|2| 3\rangle$ is connected to $\left\langle\hat{1}|2| 3 \imath_{+} 3\right\rangle$, i.e., $A R_{5} \sim A R_{9}$.

Proof. For an ellipsoid pair with modified index sequence $\langle\hat{1}|2| 3|2| 3\rangle$, the signs of the eigenvalues of the matrix $A^{-1} B$ are $\varepsilon_{1}=\varepsilon_{2}=\varepsilon_{4}=1, \varepsilon_{3}=-1$. Let the four roots of $f(\lambda)=0$ be $0<\lambda_{1}<\lambda_{2}<\lambda_{3}<\lambda_{4}$. Consider the moving quadratic pair with

$$
\begin{gathered}
A(t)=\operatorname{diag}(1,1,-1,1-t), \\
B(t)=\left(\begin{array}{cccc}
\lambda_{1} & & & \\
& \lambda_{2} & & \\
& & -\lambda_{3} & t \\
& & & \lambda_{4}
\end{array}\right),
\end{gathered}
$$


Table 3: The connection diagram of all arrangements. A $\checkmark$ means that the arrangements in the corresponding row and column are connected.

\begin{tabular}{|l|l|l|l|l|l|l|l|l|l|l|l|l|l|l|l|l|l|l|l|l|l|}
\hline cases & 1 & 2 & 3 & 4 & 5 & 6 & 7 & 8 & 9 & 10 & 11 & 12 & 13 & 14 & 15 & 16 & 17 & 18 & 19 & 20 & 21 \\
\hline 1 & & & & & & & & & & & & & $\checkmark$ & & & & & & & \\
\hline 2 & & & & & & & & & & & $\checkmark$ & & & & & & $\checkmark$ & & $\checkmark$ & & \\
\hline 3 & & & & & & & & & & & & $\checkmark$ & & & & & & $\checkmark$ & & $\checkmark$ & \\
\hline 4 & & & & & & & $\checkmark$ & & & $\checkmark$ & & & & & $\checkmark$ & $\checkmark$ & $\checkmark$ & & $\checkmark$ & $\checkmark$ & $\checkmark$ \\
\hline 5 & & & & & & & & $\checkmark$ & $\checkmark$ & & & & & $\checkmark$ & & $\checkmark$ & & $\checkmark$ & $\checkmark$ & $\checkmark$ & $\checkmark$ \\
\hline 6 & & & & & & & $\checkmark$ & $\checkmark$ & $\checkmark$ & $\checkmark$ & $\checkmark$ & $\checkmark$ & $\checkmark$ & $\checkmark$ & $\checkmark$ & $\checkmark$ & $\checkmark$ & $\checkmark$ & $\checkmark$ & $\checkmark$ & $\checkmark$ \\
\hline 7 & & & & $\checkmark$ & & $\checkmark$ & & & & & & & & & $\checkmark$ & $\checkmark$ & & & $\checkmark$ & $\checkmark$ & $\checkmark$ \\
\hline 8 & & & & & $\checkmark$ & $\checkmark$ & & & & & & & & $\checkmark$ & & $\checkmark$ & & & $\checkmark$ & $\checkmark$ & $\checkmark$ \\
\hline 9 & & & & & $\checkmark$ & $\checkmark$ & & & & & & & & $\checkmark$ & & & & $\checkmark$ & & $\checkmark$ & $\checkmark$ \\
\hline 10 & & & & $\checkmark$ & & $\checkmark$ & & & & & & & & & $\checkmark$ & & $\checkmark$ & & $\checkmark$ & & $\checkmark$ \\
\hline 11 & & $\checkmark$ & & & & $\checkmark$ & & & & & & & & & & & $\checkmark$ & & $\checkmark$ & & \\
\hline 12 & & & $\checkmark$ & & & $\checkmark$ & & & & & & & & & & & & $\checkmark$ & & $\checkmark$ & \\
\hline 13 & $\checkmark$ & & & & & $\checkmark$ & & & & & & & & & & & & & & & \\
\hline 14 & & & & & $\checkmark$ & $\checkmark$ & & $\checkmark$ & $\checkmark$ & & & & & & & & & & & $\checkmark$ & $\checkmark$ \\
\hline 15 & & & & $\checkmark$ & & $\checkmark$ & $\checkmark$ & & & $\checkmark$ & & & & & & & & & $\checkmark$ & & $\checkmark$ \\
\hline 16 & & & & $\checkmark$ & $\checkmark$ & $\checkmark$ & $\checkmark$ & $\checkmark$ & & & & & & & & & & & $\checkmark$ & $\checkmark$ & \\
\hline 17 & & $\checkmark$ & & $\checkmark$ & & $\checkmark$ & & & & $\checkmark$ & $\checkmark$ & & & & & & & & $\checkmark$ & & \\
\hline 18 & & & $\checkmark$ & & $\checkmark$ & $\checkmark$ & & & $\checkmark$ & & & $\checkmark$ & & & & & & & & $\checkmark$ & \\
\hline 19 & & $\checkmark$ & & $\checkmark$ & $\checkmark$ & $\checkmark$ & $\checkmark$ & $\checkmark$ & & $\checkmark$ & $\checkmark$ & & & & $\checkmark$ & $\checkmark$ & $\checkmark$ & & & & \\
\hline 20 & & & $\checkmark$ & $\checkmark$ & $\checkmark$ & $\checkmark$ & $\checkmark$ & $\checkmark$ & $\checkmark$ & & & $\checkmark$ & & $\checkmark$ & & $\checkmark$ & & $\checkmark$ & & & \\
\hline 21 & & & & $\checkmark$ & $\checkmark$ & $\checkmark$ & $\checkmark$ & $\checkmark$ & $\checkmark$ & $\checkmark$ & & & & $\checkmark$ & $\checkmark$ & & & & \\
\hline
\end{tabular}

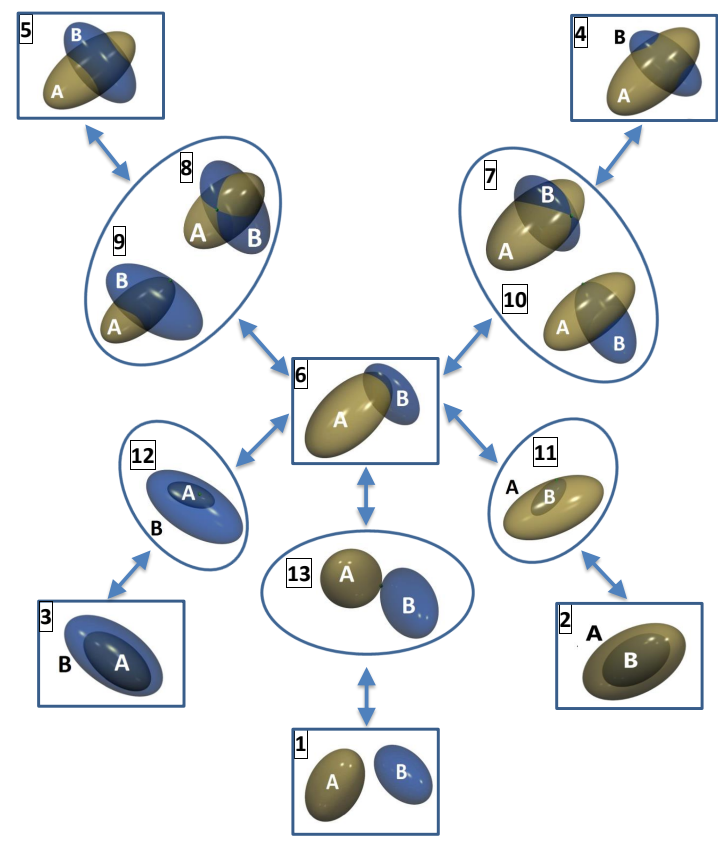

Fig. 12. Connection diagram of arrangements with non-singular intersection curve (from $A R_{1}$ to $A R_{6}$, bounded by boxes) and arrangements with one double singularity on the intersection curve (from $A R_{7}$ to $A R_{13}$, bounded by ellipses).

Then

$$
A(t)^{-1} B(t)=\left(\begin{array}{cccc}
\lambda_{1} & & & \\
& \lambda_{2} & & \\
& & \lambda_{3} & -t \\
& & & \lambda_{4} /(1-t)
\end{array}\right)
$$

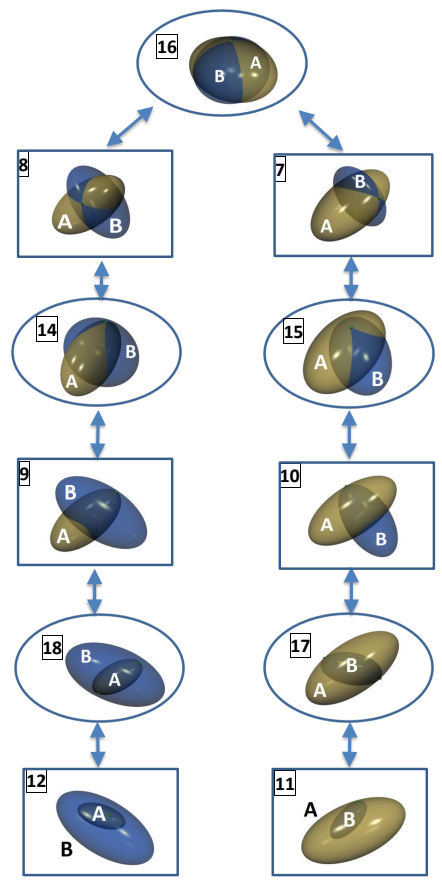

Fig. 13. Connection diagram of arrangements with one double singularity on the intersection curve (from $A R_{7}$ to $A R_{13}$, bounded by boxes) and arrangements with one triple singularity or two double singularities on the intersection curve) (from $A R_{14}$ to $A R_{18}$, bounded by ellipses).

Within $1-\frac{\lambda_{4}}{\lambda_{3}}<t<1$, the matrix $A(t)^{-1} B(t)$ has the Jordan form

$$
\left(\begin{array}{llll}
\lambda_{1} & & & \\
& \lambda_{2} & & \\
& & \lambda_{3} & \\
& & & \lambda_{4} /(1-t)
\end{array}\right)
$$




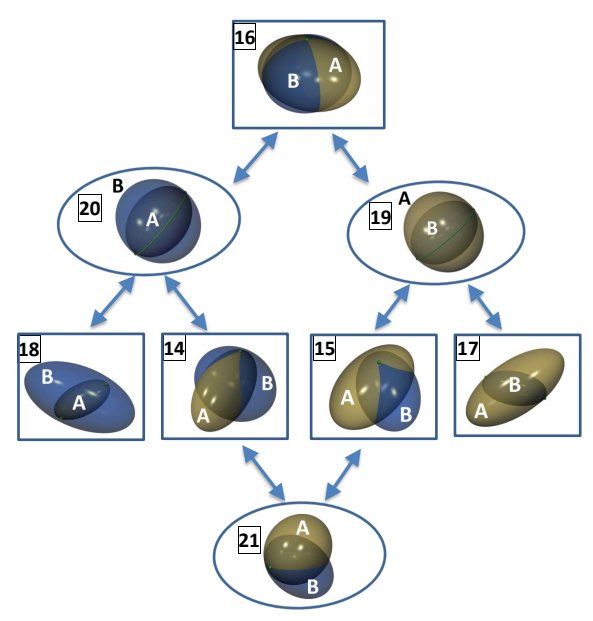

Fig. 14. Connection diagram of arrangements with one triple singularity or two double singularities on the intersection curve (from $A R_{14}$ to $A R_{18}$, bounded by boxes) and arrangements with the whole singular intersection curve (from $A R_{19}$ to $A R_{21}$, bounded by ellipses).

while at $t=1-\frac{\lambda_{4}}{\lambda_{3}}$, the Jordan form of $A(t)^{-1} B(t)$ suddenly changes to

$$
\left(\begin{array}{cccc}
\lambda_{1} & & & \\
& \lambda_{2} & & \\
& & \lambda_{3} & 1 \\
& & & \lambda_{3}
\end{array}\right),
$$

this proves the connection $A R_{5} \sim A R_{9}$.

The output of the classification algorithm depends on the computation of the sign of algebraic expressions of low degree of the input coefficients of the two quadrics $A$ and $B$. If these coefficients are given exactly (e.g. as rational numbers), the algorithm is able to determine exactly the arrangement type. If the computation is performed with approximate coefficients, the classification is stable when the input is far enough from the boundary of the intersection morphology region. Nearby the boundaries, where signs cannot be guaranteed for the approximate input, the connection diagram between the $\mathrm{d}$ ifferent morphologies allows us to determine which are the plausible ones.

\section{CONCLUSIONS}

We give a full classification and enumeration of the arrangements formed by two ellipsoids and provide an efficient algorithm of deciding the arrangement of two given ellipsoids. The algebraic conditions for characterizing the arrangements are modified index sequences, which are the main ingredients behind the classification, enumeration as well as the decision algorithm for arrangements. The decision of the arrangement of two given ellipsoids can be done efficiently in $O(1)$ arithmetic operations. A stratification diagram showing the connections of all enumerated arrangements is also built, which indicates intrinsic connections among these enumerated arrangements.

The strategy of using modified index sequences can be expected to be extended to two dimensional cases for characterizing the arrangements of two ellipses. Another possible direction of extension is to study the arrangements of two ellipsoids also in higher dimensions. The continuous detection of the variations of the arrangements of two ellipsoids is also a topic for further exploration. These extensions can be our future work.

\section{SUPPLEMENTARY MATERIALS}

See the supplementary materials for the preparations of Jordan forms, Segre characteristics and quadratic canonical forms (Section 1 in the appendix), proofs of the enumerations of arrangements (Section 2 in the appendix) and the connection diagrams of arrangements (Section 3 in the appendix) in the online version. The reader may also follow here ${ }^{2}$ to access the full paper with all the proofs.

\section{ACKNOWLEDGMENTS}

The work is supported by the National Natural Science Foundation of China (61872354, 61772318), the Beijing Natural Science Foundation (Z190004), the Intelligent Science and Technology Advanced subject project of University of Chinese Academy of Sciences (115200S001), and the Alibaba Group through Alibaba Innovative Research Program.

\section{REFERENCES}

P. K. Agarwal, M. d. Berg, J. Matousek, and O. Schwarzkopf. 1998. Constructing Levels in Arrangements and Higher Order Voronoi Diagrams. SIAM J. Comput. 27, 3 (1998), 654-667.

M. Alberich-Carraminana, B. Elizalde, and F. Thomas. 2017. New algebraic conditions for the identification of the relative position of two coplanar ellipses. Computer Aided Geometric Design 54 (2017), $35-48$.

M. d. Berg, O. Cheong, M. v. Kreveld, and M. Overmars. 2013. Computational geometry: Algorithms and Applications (3 ed.). Springer.

T. J. I'A. Bromwich. 1960. Quadratic forms and their classification by means of invariant-factors. Cambridge tracts in mathematics and mathematical physics, Vol. 3. Hafner, New York.

Jung-Woo Chang, Wenping Wang, and Myung-Soo Kim. 2008. Efficient Collision Detection Using a Dual Bounding Volume Hierarchy. In Advances in Geometric Modeling and Processing. Springer Berlin Heidelberg, Berlin, Heidelberg, 143-154.

Y.-K. Choi. 2008. Collision Detection for Ellipsoids and Other Quadrics. Ph.D. Dissertation. The University of Hong Kong, Pokfulam Road, Hong Kong.

Y.-K. Choi, J.-W. Chang, W. Wang, M.-S. Kim, and G. Elber. 2009. Continuous Collision Detection for Ellipsoids. IEEE Transactions on Visualization and Computer Graphics 15, 2 (2009), 311-325.

Y.-K. Choi, W. Wang, Y. Liu, and M.-S. Kim. 2006. Continuous Collision Detection for Two Moving Elliptic Disks. IEEE Transactions on Robotics 22, 2 (2006), 213-224.

Alexandru Dimca. 2017. Hyperpane Arrangements - An Introduction. Springer.

L. Dupont, D. Lazard, S. Lazard, and S. Petitjean. 2008a. Nearoptimal parameterization of the intersection of quadrics: I. The generic algorithm. Journal of Symbolic Computation 43, 3 (2008), $168-191$.

L. Dupont, D. Lazard, S. Lazard, and S. Petitjean. 2008b. Near-optimal parameterization of the intersection of quadrics: II. A classification

\footnotetext{
${ }^{2}$ http://www.mmrc.iss.ac.cn/ xhjia/EllipArrange.pdf
} 
of pencils. Journal of Symbolic Computation 43, 3 (2008), 192 215.

L. Dupont, D. Lazard, S. Lazard, and S. Petitjean. 2008c. Near-optimal parameterization of the intersection of quadrics: III. Parameterizing singular intersections. Journal of Symbolic Computation 43, 3 (2008), $216-232$.

D. H. Eberly. 2001. 3D Game Engine Design. Academic Press.

F. Etayo, L. Gonzalez-Vega, and N. del Rio. 2006. A new approach to characterizing the relative position of two ellipses depending on one parameter. Computer Aided Geometric Design 23 (2006), 324-350.

R. T. Farouki, C. Neff, and M. A. O'Conner. 1989. Automatic parsing of degenerate quadric-surface intersections. ACM Transactions on Graphics 8, 3 (1989), 174-203.

Stefan Felsner. 2004. Geometric Graphs and Arrangements. Springer.

E. Ghossein and M. Lévesque. 2013. Random generation of periodic hard ellipsoids based on molecular dynamics: A computationallyefficient algorithm. J. Comput. Phys. 253 (2013), $471-490$.

A. A. Goncalves, A. Bernardino, J. Jorge, and D. S. Lopes. 2017. A benchmark study on accuracy-controlled distance calculation between superellipsoid and superovoid contact geometries. Mechanism and Machine Theory 115 (2017), 77 - 96.

S. Gottschalka, C. L. Ming, and D. Manocha. 1996. OBBTree: A hierarchical structure for rapid interference detection. Proceedings of Siggraph (1996), 171-180.

D. Halperin. 2004. Arrangements. Handbook of Discrete \& Computational Geometry 21, 4 (2004), 389-412.

D Halperin and C. R Shelton. 1997. A Perturbation Scheme for Spherical Arrangements with Application to Molecular Modeling. Computational Geometry 10, 4 (1997), 273-287.

P. M. Hubbard. 1996. Approximating polyhedra with spheres for timecritical collision detection. ACM Transactions on Graphics 15, 3 (1996), 179-210.

X. Jia, Y.-K. Choi, B. Mourrain, and W. Wang. 2011. An algebraic approach to continuous collisiondetection for ellipsoids. Computer Aided Geometric Design 28, 3 (2011), 164-176.

M. Ju, J. Liu, S. Shiang, Y. Chien, K. Hwang, and W. Lee. 2001. A novel collision detection method based on enclosed ellipsoid. In Proceedings of 2001 IEEE Conference on Robotics and Automation. 21-26.

J. T. Klosowski, M. Held, J. S. B. Mitchell, H. Sowizral, and K. Zikan. 2002. Efficient collision detection using bounding volume hierarchies of k-DOPs. IEEE Transactions on Visualization \& Computer Graphics 4, 1 (2002), 21-36.

B. H. Lee, Jae D. Jeon, and Jung H. Oh. 2017. Velocity obstacle based local collision avoidance for a holonomic elliptic robot. Autonomous Robots 41, 6 (01 Aug 2017), 1347-1363.

J. Z. Levin. 1979. Mathematical models for determining the intersections of quadric surfaces. Comput. Graph. Image Process. 1 (1979), $73-87$.

S. Liang, C. Li, X. Guo, B. Prabhakaran, and J. Chai. 2016. Motion Capture With Ellipsoidal Skeleton Using Multiple Depth Cameras. IEEE Transactions on Visualization \& Computer Graphics PP, 99 (2016), 1-1.

S. Liu, C. L. Wang, K. C. Hui, X. Jin, and H. Zhao. 2007. Ellipsoid-tree construction for solid objects. In ACM Symposium on Solid and Physical Modeling. 303-308.

L. Lu, Y.-K. Choi, W. Wang, and M.-S. Kim. 2007. Variational 3D Shape Segmentation for Bounding Volume Computation. In Computer Graphics Forum. 329-338.

J. R. Miller. 1987. Geometric Approaches to Nonplanar Quadric Surface Intersection Curves. ACM Transactions on Graphics 6, 4 (1987), 274-307.

J. R. Miller and R. N. Goldman. 1995. Geometric Algorithms for Detecting and Calculating All Conic Sections in the Intersection of Any Two Natural Quadric Surfaces. CVGIP: Graphical Model and Image Processing 57, 1 (1995), 55-66.

B. Mirtich. 1998. V-Clip: Fast and Robust Polyhedral Collision Detection. ACM Transactions on Graphics 17, 3 (July 1998), 177-208.

I. J. Palmer and R. L Grimsdale. 1995. Collision Detection for Animation using Sphere-trees. In Computer Graphics Forum. 105-116.

E. Rimon and S. Boyd. 1977. Obstacle collision detection using best ellipsoid fit. J. Intelligent Robotics Syst. 18 (1977), 105-126.

S. M. Rubio-Largo, P. G. Lind, D. Maza, and R. C. Hidalgo. 2015. Granular gas of ellipsoids: analytical collision detection implemented on GPUs. Computational Particle Mechanics 2, 2 (01 Jun 2015), $127-138$.
A. Schweikard, J. R. Adler, and J. Latombe. 1992. Motion planning in stereotaxic radiosurgery. IEEE Transactions on Robotics and Automation 9, 6 (1992), 764-774.

C. Shene and J. Johnstone. 2014. On the lower degree intersections of two natural quadrics. ACM Transactions on Graphics 13, 4 (2014), $400-424$.

S. P. Shiang, J. S. Liu, and Y. R. Chien. 2000. Estimate of minimum distance between convex polyhedra based on enclosed ellipsoids. In Proceedings of the IEEE/RST International Conference on Intelligent Robots and Systems, Takamatsu, Japan, October. 739744.

M. Tang, R. Tong, Z. Wang, and D. Manocha. 2014. Fast and Exact Continuous Collision Detection with Bernstein Sign Classification. ACM Trans. Graph. 33, 6, Article 186 (Nov. 2014), 8 pages.

C. Tu, W. Wang, B. Mourrain, and J. Wang. 2009. Using signature sequences to classify intersection curves of two quadrics. Computer Aided Geometric Design 26 (2009), 317 - 335.

H. Wang. 2014. Defending Continuous Collision Detection Against Errors. ACM Transactions on Graphics 33, 4 (2014), 122:1-122:10.

W. Wang. 2002. Modeling and Processing with Quadric Surfaces. In Handbook of Computer Aided Geometric Design, M.S. Kim G. Farin, J. Hoschek (Ed.). Elsevier, 777-795.

W. Wang, Y.-K. Choi, B. Chan, M.-S. Kim, and J. Wang. 2004. Efficient Collision Detection for Moving Ellipsoids Using Separating Planes. Computing 72, 1-2 (2004), 235-246.

W. Wang, J. Wang, and M.-S. Kim. 2001. An algebraic condition for the separation of two ellipsoids. Computer Aided Geometric Design 18,6 (2001), 531-539.

Y. Xu and S. Chen. 2016. A method for modeling the damage behavior of concrete with a three-phase mesostructure. Construction and Building Materials 102 (2016), 26 - 38. 


\title{
Complete Classification and Efficient Determination of Arrangements Formed by Two Ellipsoids - Appendix
}

\author{
XIAOHONG JIA, KLMM, AMSS, Chinese Academy of Sciences \& University of Chinese Academy of Science, China \\ CHANGHE TU, School of Computer Science and Technology, Shandong University, China \\ BERNARD MOURRAIN, Aromath, Inria Méditerranée, Université Côte d'Azur, France \\ WENPING WANG, University of Hong Kong, Hong Kong
}

ACM Reference Format:

Xiaohong Jia, Changhe Tu, Bernard Mourrain, and Wenping Wang. 2020. Complete Classification and Efficient Determination of Arrangements Formed by Two Ellipsoids - Appendix. ACM Trans. Graph. 39, 3, Article 27 (May 2020), 8 pages. https: //doi.org/10.1145/nnnnnnn.nnnnnnn

\section{JORDAN FORMS, SEGRE CHARACTERISTICS AND QUADRATIC CANONICAL FORMS}

We review necessary knowledge about Jordan forms, Segre characteristics and quadratic canonical forms. Readers can find related materials in [Tu et al. 2009; Uhlig 1976; Williamson 1935].

Two matrices $M$ and $N$ from $\mathbb{R}^{k \times k}$ are said to be similar if there is a nonsingular matrix $P$ such that $M=P^{-1} N P$. Each matrix $A \in \mathbb{R}^{k \times k}$ is similar to its Jordan normal form, which shall be reviewed below.

Definition 1.1. A $k \times k$ square matrix of the form

$$
M=\left(\begin{array}{cccc}
\lambda & e & & \\
& \cdot & \cdot & \\
& & \cdot & e \\
& & & \lambda
\end{array}\right)_{k \times k}
$$

is called a Jordan block of type $I$ associated with $\lambda$ if $\lambda \in \mathbb{R}$ and $e=1$ for $k \geq 2$ or $M=(\lambda)$ with $\lambda \in \mathbb{R}$ for $k=1 ; M$ is called a Jordan form of type $I I$ associated with complex conjugate values $a \pm i b$ if

$$
\lambda=\left(\begin{array}{cc}
a & -b \\
b & a
\end{array}\right) a, b \in \mathbb{R}, b \neq 0 \text { and } e=\left(\begin{array}{ll}
1 & 0 \\
0 & 1
\end{array}\right)
$$

for $k \geq 4$ or

$$
M=\left(\begin{array}{cc}
a & -b \\
b & a
\end{array}\right)
$$

for $k=2$, with $a, b \in \mathbb{R}, b \neq 0$.

Authors' addresses: Xiaohong Jia, KLMM, AMSS, Chinese Academy of Sciences \& University of Chinese Academy of Science, Beijing, 100190 China, xhjia@amss.ac.cn; Changhe Tu, School of Computer Science and Technology, Shandong University, Qingdao, 266237, China, chtu@sdu edu.cn; Bernard Mourrain, Aromath, Inria Méditerranée, Université Côte d'Azur, 06902, France, Bernard.Mourrain@inria.fr; Wenping Wang, University of Hong Kong, Department of Computer Science, Hong kong Hong Kong, wenping@cs.hku.hk.

(C) 2020 Association for Computing Machinery.

This is the author's version of the work. It is posted here for your personal use. Not for redistribution. The definitive Version of Record was published in ACM Transactions on Graphics, https://doi.org/10. 1145/nnnnnnn.nnnnnnn.
Definition 1.2. For any matrix $A \in \mathbb{R}^{k \times k}$, there exists a quasi-diagonal matrix $J$

$$
J=\left(\begin{array}{cccc}
C\left(\lambda_{1}\right) & & & \\
& C\left(\lambda_{2}\right) & & \\
& & \ddots & \\
& & & C\left(\lambda_{k}\right)
\end{array}\right)
$$

similar to $A$, where

$$
C\left(\lambda_{i}\right)=\left(\begin{array}{cccc}
J_{1}^{(i)} & & & \\
& J_{2}^{(i)} & & \\
& & \ddots & \\
& & & J_{k_{i}}^{(i)}
\end{array}\right)
$$

for which $J_{1}^{(i)}, \cdots, J_{k_{i}}^{(i)}$ are all Jordan blocks (of type I or type II) associated with the same eigenvalue $\lambda_{i}$ of the matrix $A$. The quasi-diagonal matrix $J$ is called the Jordan normal form of the matrix $A$, and the blocks $C\left(\lambda_{i}\right)$ are called the full Jordan chain associated with the eigenvalue $\lambda_{i}, i=1, \cdots, k$. The Jordan normal form is unique up to permutation of the Jordan blocks.

Definition 1.3. The Segre characteristic of the quadric pencil $\lambda A-B$ is the integer chain of the orders of the blocks in the Jordan normal form of the matrix $A^{-1} B$, with those integers corresponding to blocks containing the same eigenvalue bracketed together, and the number of distinct real eigenvalues of the matrix $A^{-1} B$ as the subscript. For example, if the Jordan form of the matrix $A^{-1} B$ is

$$
\left(\begin{array}{cccc}
\alpha & 1 & & \\
& \alpha & & \\
& & \alpha & \\
& & & \beta
\end{array}\right)
$$

where $\alpha, \beta$ are real numbers, the Segre characteristic of the quadric pencil $\lambda A-B$ is $[(21) 1]_{2}$. In this example we also say that the Segre characteristic of $\lambda A-B$ associated with the root $\alpha$ is [21], and the Segre characteristic of $\lambda A-B$ associated with the $\operatorname{root} \beta$ is [1].

Lemma 1.4. (Quadric Pair Canonical Form). Let $A$ and $B$ be a nonsingular pair of real symmetric matrices of size $n$. Suppose that $A^{-1} B$ has real Jordan normal form

$$
\operatorname{diag}\left(J_{1}, \ldots, J_{r}, J_{r+1}, \ldots, J_{m}\right),
$$

where $J_{1} \ldots, J_{r}$ are Jordan blocks of type $I$ corresponding to the real eigenvalues of $A^{-1} B$ and $J_{r+1}, \ldots, J_{m}$ are Jordan 
blocks of type II corresponding to the complex eigenvalues of $A^{-1} B$. Then $A$ and $B$ are simultaneously congruent by a real congruence transformation to

$$
A^{\prime}=\operatorname{diag}\left(\varepsilon_{1} E_{1}, \ldots, \varepsilon_{r} E_{r}, E_{r+1}, \ldots, E_{m}\right)
$$

and

$$
B^{\prime}=\operatorname{diag}\left(\varepsilon_{1} E_{1} J_{1}, \ldots, \varepsilon_{r} E_{r} J_{r}, E_{r+1} J_{r+1}, \ldots, E_{m} J_{m}\right),
$$

respectively, where $\varepsilon_{i}= \pm 1$ and the $E_{i}$ are of the form

$$
\left(\begin{array}{cccc}
0 & \cdot & 0 & 1 \\
\cdot & \cdot & 1 & \cdot \\
\cdot & 1 & \cdot & \cdot \\
1 & 0 & \cdot & 0
\end{array}\right)
$$

of the same size as $J_{i}, i=1,2, \ldots, m$. The signs of $\varepsilon_{i}$ are unique (up to permutations) for each set of indices $i$ that are associated with a set of identical Jordan blocks $J_{i}$ of type $I$.

\section{SIGNATURE AND JORDAN FORMS}

In this section, we explain in detail the strategy of Step 7 in Algorithm 1, i.e., how to decide the Jordan blocks of matrix $A^{-1} B$ associated to its eigenvalues $\lambda_{i}, i=1, \cdots, r$, which are also roots of the characteristic function $f(\lambda)=0$.

Definition 2.1. For a fixed value $\lambda$, the signature of the matrix $\lambda A-B$ is the pair of integers $(p, n)$, where $p$ and $n$ are the number of positive and negative eigenvalues of the matrix $\lambda A-B$.

Let $C(\lambda, \mu)=\operatorname{det}(\lambda A-B-\mu I)=c_{0}(\lambda)+c_{1}(\lambda) \mu+c_{2}(\lambda) \mu^{2}+$ $c_{3}(\lambda) \mu^{3}+\mu^{4}$ be the eigencurve of the ellipsoid pencil. Then the signature of $\lambda A-B$ can be decided as follows.

LEMma 2.2. For any $\lambda \in \mathbb{R}$, the number of positive eigenvalues of the matrix $\lambda A-B$ is the number of sign variations of $\left[c_{0}(\lambda), c_{1}(\lambda), c_{2}(\lambda), c_{3}(\lambda), 1\right]$; the number of negative eigenvalues of the matrix $\lambda A-B$ is the number of sign variations of $\left[c_{0}(\lambda),-c_{1}(\lambda), c_{2}(\lambda),-c_{3}(\lambda), 1\right]$.

In step 7 of Algorithm 1, one needs to decide the Jordan blocks of the matrix $A^{-1} B$ associated to its eigenvalue $\lambda=\lambda_{i}$, where $\lambda_{i}$ is also a root of the characteristic polynomial $f(\lambda)=$ $c_{0}(\lambda)=0, i=1, \cdots, r$. This is achieved by computing the signature $\left(p_{i}, n_{i}\right)$ of the matrix $\lambda_{i} A-B$. Hence $\left(p_{i}, n_{i}\right)$ can be computed by the above lemma. However, since the root $\lambda_{i}$ is generically not rational hence represented by an isolated interval $\left[a_{i}, b_{i}\right]$, the signs of $c_{j}\left(\lambda_{i}\right), j=1,2,3$ are computed by $V_{c_{0}, c_{j}}\left(a_{i}\right)-V_{c_{0}, c_{j}}\left(b_{i}\right)$, where $V_{c_{0}, c_{j}}$ means the number of sign variations of the sub-resultant sequence of $c_{0}(\lambda)$ and $c_{0}^{\prime} c_{j}(\lambda)$.

Once $\left(p_{i}, n_{i}\right)$ are computed, the Jordan block associated to the eigenvalue $\lambda_{i}$ can be got from Table 1. For example, if we have known that $\lambda_{i}$ is a triple root (this can be done in the root isolation step), we just need to first check the value of $p_{i}+n_{i}$ from the table. If $p_{i}+n_{i}=1$, we directly know that the Jordan blocks associate to $\lambda_{i}$ are \|\| ; if $p_{i}+n_{i}=3$, we know that the Jordan block is $\varkappa_{ \pm}$; if $p_{i}+n_{i}=2$, we further
Table 1. Correspondence between signature and Jordan forms

\begin{tabular}{|c|c|c|c|c|}
\hline Multi & Jordan & $p_{i}+n_{i}$ & $p_{i}-s_{i-1}$ & $s_{i}-p_{i}$ \\
\hline Simple root & $\mid$ & 3 & - & - \\
\hline \multirow{3}{*}{ double root } & $\|$ & 2 & - & - \\
\cline { 2 - 5 } & $\varkappa_{+}$ & 3 & -1 & 1 \\
\cline { 2 - 5 } & $\varkappa_{-}$ & 3 & 0 & 0 \\
\hline \multirow{3}{*}{ triple root } & $\varkappa_{ \pm}$ & 3 & - & - \\
\cline { 2 - 5 } & $\varkappa_{+} \mid$ & 2 & -1 & 2 \\
\cline { 2 - 5 } & $\varkappa_{-} \mid$ & 2 & 0 & 1 \\
\cline { 2 - 5 } & $\mid \|$ & 1 & - & - \\
\hline \multirow{3}{*}{ quadruple root } & $\varkappa_{ \pm} \mid$ & 2 & - & - \\
\cline { 2 - 6 } & $\varkappa_{+} \|$ & 1 & -1 & 3 \\
\cline { 2 - 6 } & $\varkappa_{-} \|$ & 1 & 0 & 2 \\
\hline
\end{tabular}

examine the value of $p_{i}-s_{i-1}$ and $s_{i}-p_{i}$, where $s_{i}=\operatorname{Id}\left(q_{i}\right)$ and $q_{i}$ are the rational values separating the roots, i.e.,

$$
-\infty<\lambda_{1}<q_{1}<\lambda_{2}<\cdots<q_{r-1}<\lambda_{r}<+\infty
$$

and $s_{0}=\operatorname{Id}(-\infty), s_{r}=\operatorname{Id}(+\infty)$. Then we know whether the Jordan blocks are $\imath_{-}$or $\imath_{+} \mid$. Note that Table 1 is summarized from results of [Tu et al. 2009].

\section{PROOF OF THE ARRANGEMENT ENUMERATION}

We have shown in Theorem 5.1 the five possible non-equivalent arrangements corresponding to the five different modified index sequences when the characteristic polynomial has four different real roots and the Segre characteristic is $[1111]_{4}$. Next we analyze other arrangements corresponding to more complex situations of the roots of characteristic polynomial that are shown in Table 1(a)-(c). Note that in each of the following theorems, the enumeration of the modified index sequence for each Segre characteristic is based on the rule that $\operatorname{Id}(0)=1, \operatorname{Id}(-\infty)=1$ and $\operatorname{Id}(+\infty)=3$ as shown in Theorem 5.1. We will not repeat this detail every time and give only the analysis on the arrangement for each of these enumerated modified index sequences.

Theorem 3.1. Given a pair of ellipsoids $\mathcal{A}$ and $\mathcal{B}$, if the characteristic polynomial has two complex roots, then the Segre characteristic of the pencil $\lambda A-B$ can only be $[1111]_{2}$ or $[(11) 11]_{1}$, corresponding to the following two modified index sequences.

(1) $\langle\hat{1}|2| 3\rangle-\mathcal{A}$ and $\mathcal{B}$ intersect in a non-singular loop (case $6)$

(2) $\langle\hat{1} \| 3\rangle-\mathcal{A}$ and $\mathcal{B}$ intersect in a planar loop (case 24); Proof.

(1) If the index sequence is $\langle\hat{1}|2| 3\rangle$, by [Tu et al. 2009], the intersection curve of the two ellipsoids is a non-singular loop. The two ellipsoids are partitioned by each other in a symmetric manner, hence only one arrangement is possible.

(2) If the index sequence is $\langle\hat{1}|| 3\rangle$, the intersection curve has two possible morphologies, but only one is closed hence possible for two ellipsoids, whose real part is a 
planar loop. Also the two ellipsoids partition each other in a symmetric manner, hence only one arrangement is possible.

Theorem 3.2. Given a pair of ellipsoids $\mathcal{A}$ and $\mathcal{B}$, if the characteristic polynomial has a double real root and two other simple real roots with the Segre characteristic $[211]_{3}$, then all possible corresponding index sequences and arrangements are as follows.

(1) $\left\langle\hat{1}\left|2 \imath_{+} 2\right| 3\right\rangle-\mathcal{B}$ penetrates $\mathcal{A}$ and intersect with $\mathcal{A}$ at a null-homotopic component with a crunode (case 7);

(2) $\left\langle\hat{1}\left|2 \ell_{-} 2\right| 3\right\rangle-\mathcal{A}$ penetrates $\mathcal{B}$ and intersect with $\mathcal{B}$ at a null-homotopic component with a crunode (case 8);

(3) $\left\langle\hat{1}|2| 3 \imath_{+} 3\right\rangle-$ part of $\mathcal{A}$ goes inside $\mathcal{B}$ and touch $\mathcal{B}$ internally at an acnode (case 9);

(4) $\left\langle\hat{1} \imath_{-} 1|2| 3\right\rangle$ - part of $\mathcal{B}$ goes inside $\mathcal{A}$ and touch $\mathcal{A}$ internally at an acnode (case 10);

(5) $\left\langle\hat{1} \imath_{+} 1|2| 3\right\rangle-\mathcal{B}$ is inside $\mathcal{A}$ and touches $\mathcal{A}$ at an acnode (case 11);

(6) $\left\langle\hat{1}|2| 3 l_{-} 3\right\rangle-\mathcal{A}$ is inside $\mathcal{B}$ and touches $\mathcal{B}$ at an acnode (case 12);

(7) $\left\langle 1 \chi_{ \pm} \hat{1}|2| 3\right\rangle-\mathcal{A}$ and $\mathcal{B}$ touch externally at an acnode (case 13).

Proof. We now suppose that the double root is $\lambda_{0}$ and the other two simple roots are $\lambda_{1}<\lambda_{2}$, and the $\operatorname{sign} \varepsilon_{i}$ is for $\lambda_{i}$, $i=0,1,2$.

(1) If the index sequence is $\left\langle\hat{1}\left|2 \imath_{+} 2\right| 3\right\rangle$, then $0<\lambda_{1}<\lambda_{0}<$ $\lambda_{2}$ and $\varepsilon_{0}=\varepsilon_{1}=\varepsilon_{2}=1$. By Lemma 1.4, the canonical forms of $\mathcal{A}$ and $\mathcal{B}$ are

$A=\left(\begin{array}{cccc}1 & 0 & 0 & 0 \\ 0 & 0 & 1 & 0 \\ 0 & 1 & 0 & 0 \\ 0 & 0 & 0 & 1\end{array}\right), B=\left(\begin{array}{cccc}\varepsilon_{1} \lambda_{1} & 0 & 0 & 0 \\ 0 & 0 & \varepsilon_{0} \lambda_{0} & 0 \\ 0 & \varepsilon_{0} \lambda_{0} & \varepsilon_{0} & 0 \\ 0 & 0 & 0 & \varepsilon_{2} \lambda_{2}\end{array}\right)$.

Letting $y=\frac{\bar{y}+\bar{z}}{\sqrt{2}}, z=\frac{\bar{y}-\bar{z}}{\sqrt{2}}$, the two ellipsoids $\mathcal{A}$ and $\mathcal{B}$ are transformed to

$$
\begin{aligned}
& \mathcal{A}^{\prime}=x^{2}+\bar{y}^{2}-\bar{z}^{2}+w^{2}=0 \\
& \mathcal{B}^{\prime}=\lambda_{1} x^{2}+\left(\lambda_{0}+\frac{1}{2}\right) \bar{y}^{2}-\left(\lambda_{0}-\frac{1}{2}\right) \bar{z}^{2}+\lambda_{2} w^{2}-\bar{y} \bar{z}=0 .
\end{aligned}
$$

Choose $\bar{z}=0$ as the plane at infinity. Then $\mathcal{A}^{\prime}$ is a unit sphere and $\mathcal{B}^{\prime}$ has the equation

$$
\frac{\lambda_{1}}{\lambda_{0}+\frac{1}{2}} x^{2}+\left(\bar{y}-\frac{1}{2 \lambda_{0}+1}\right)^{2}+\frac{\lambda_{2}}{\lambda_{0}+\frac{1}{2}} w^{2}=\frac{\lambda_{0}^{2}}{\left(\lambda_{0}+\frac{1}{2}\right)^{2}} .
$$

On the plane $x=0$, the section of $\mathcal{A}^{\prime}$ is a unit circle and the section of $\mathcal{B}^{\prime}$ is the ellipse

$$
\frac{\left(\bar{y}-y_{0}\right)^{2}}{a^{2}}+\frac{w^{2}}{b^{2}}=1,
$$

where $y_{0}=\frac{1}{2\left(\lambda_{0}+\frac{1}{2}\right)}, a=\frac{\lambda_{0}}{\lambda_{0}+\frac{1}{2}}, b=\frac{\lambda_{0}}{\sqrt{\left(\lambda_{0}+\frac{1}{2}\right) \lambda_{2}}}$. Note that $y_{0}+a=1$ and $a<1$. Also note that $b<\frac{\lambda_{0}}{\sqrt{\lambda_{0} \lambda_{2}}}=\sqrt{\frac{\lambda_{0}}{\lambda_{2}}}<1$. Hence the cross sections of $\mathcal{A}^{\prime}$ and $\mathcal{B}^{\prime}$ on the plane $x=0$ is the left figure of Figure 1. On the plane $w=0$, the section of

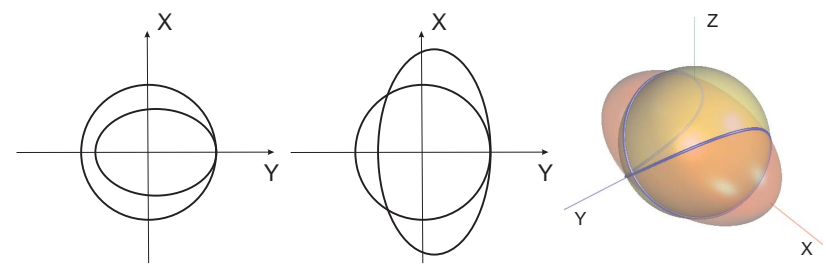

Fig. 1. Left: Plane sections of $\mathcal{A}^{\prime}$ and $\mathcal{B}^{\prime}$ on $x=0$; middle: plane sections of $\mathcal{A}^{\prime}$ and $\mathcal{B}^{\prime}$ on $w=0$; right: affine realization of $\mathcal{A}$ and $\mathcal{B}$.

$\mathcal{A}^{\prime}$ is a unit circle, and the section of $\mathcal{B}^{\prime}$ is

$$
\frac{\left(\bar{y}-y_{0}\right)^{2}}{a^{2}}+\frac{x^{2}}{b^{2}}=1 \text {, }
$$

where $y_{0}=\frac{1}{2\left(\lambda_{0}+\frac{1}{2}\right)}, a=\frac{\lambda_{0}}{\lambda_{0}+\frac{1}{2}}, b=\frac{\lambda_{0}}{\sqrt{\left(\lambda_{0}+\frac{1}{2}\right) \lambda_{1}}}$. Note that $y_{0}+a=1$ and $a<1$. Also note that $b<\frac{\lambda_{0}}{\sqrt{\lambda_{0} \lambda_{1}}}=\sqrt{\frac{\lambda_{0}}{\lambda_{1}}}>1$. Hence the cross sections of $\mathcal{A}^{\prime}$ and $\mathcal{B}^{\prime}$ on the plane $w=0$ is the middle figure of Figure 1. Hence the affine realization of the arrangement of the two ellipsoid is as in the right figure of Figure 1.

(2) If the index sequence is $\left\langle\hat{1}\left|2 \ell_{-} 2\right| 3\right\rangle$, similar treatment as above can be used by noticing that $\varepsilon_{0}=-1$.

(3) If the index sequence is $\left\langle\hat{1}|2| 3 \imath_{+} 3\right\rangle$, then $0<\lambda_{1}<\lambda_{2}<$ $\lambda_{0}$. By Lemma 1.4, $A$ and $B$ are simultaneously congruent to following Quadratic Pair Canonical Forms:

$A=\left(\begin{array}{cccc}\varepsilon_{1} & 0 & 0 & 0 \\ 0 & \varepsilon_{2} & 0 & 0 \\ 0 & 0 & 0 & \varepsilon_{0} \\ 0 & 0 & \varepsilon_{0} & 0\end{array}\right), B=\left(\begin{array}{cccc}\varepsilon_{1} \lambda_{1} & 0 & 0 & 0 \\ 0 & \varepsilon_{2} \lambda_{2} & 0 & 0 \\ 0 & 0 & 0 & \varepsilon_{0} \lambda_{0} \\ 0 & 0 & \varepsilon_{0} \lambda_{0} & \varepsilon_{0}\end{array}\right)$,

where $\varepsilon_{0}=\varepsilon_{1}=\varepsilon_{2}=1$. By setting $z=\frac{\bar{z}+\bar{w}}{\sqrt{2}}, w=\frac{\bar{z}-\bar{w}}{\sqrt{2}}$, we transform the equations of $\mathcal{A}$ and $\mathcal{B}$ to

$$
\begin{aligned}
& \mathcal{A}^{\prime}: x^{2}+y^{2}+\bar{z}^{2}-\bar{w}^{2}=0 \\
& \mathcal{B}^{\prime}: \lambda_{1} x^{2}+\lambda_{2} y^{2}+\left(\lambda_{0}+\frac{1}{2}\right) \bar{z}^{2}-\left(\lambda_{0}-\frac{1}{2}\right) \bar{w}^{2}-\bar{z} \bar{w}=0
\end{aligned}
$$

Letting $\bar{w}=0$ be the plane at infinity, then $\mathcal{A}^{\prime}$ is a unit sphere and $\mathcal{B}^{\prime}$ is an ellipsoid with the equation

$$
\frac{\lambda_{1}}{\lambda_{0}+\frac{1}{2}} x^{2}+\frac{\lambda_{2}}{\lambda_{0}+\frac{1}{2}} y^{2}+\left(\bar{z}-\frac{1}{2\left(\lambda_{0}+\frac{1}{2}\right)}\right)^{2}=\frac{\lambda_{0}^{2}}{\left(\lambda_{0}+\frac{1}{2}\right)^{2}}
$$

Similar analysis on the size of the sphere and the ellipsoid can be performed as above. The cross-sections of $\mathcal{A}^{\prime}, \mathcal{B}^{\prime}$ at the plane $y=0$ and $\bar{z}=0$ are as shown in the left and middle figures in Fig 2, and the intersection curve of $\mathcal{A}^{\prime}$ and $\mathcal{B}^{\prime}$ is a real loop and a acnode, as shown in the right figure in Fig 2.

Other cases can be analysed in a similar way. We omit details here.

Theorem 3.3. Given two ellipsoids $\mathcal{A}$ and $\mathcal{B}$, if their characteristic polynomial has a triple root and a simple root with Segre characteristic $[31]_{2}$, then they have the following two possible index sequences and corresponding arrangement:

(1) $\left\langle\hat{1} u_{+} 2 \mid 3\right\rangle-\mathcal{A}$ and $\mathcal{B}$ intersect in a singular curve with a cusp (case 14), and the arrangement is connected with 


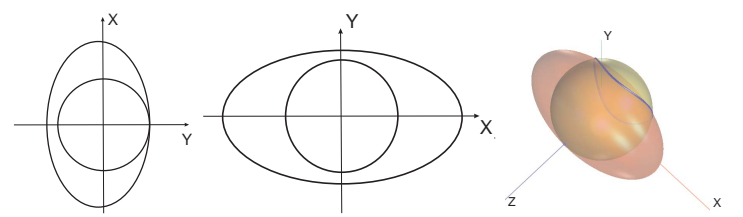

Fig. 2. Left and middle: the sectional view in the planes $y=0$ and $\bar{z}=0$; right: The arrangement of the ellipsoid $\mathcal{B}^{\prime}$ and the unit sphere $\mathcal{A}^{\prime}$.

the one in case 26 ( $\mathcal{A}$ internally touches $\mathcal{B}$ at a double conic).

(2) $\left\langle\hat{1} \mid 2 \varkappa_{+} 3\right\rangle-\mathcal{A}$ and $\mathcal{B}$ intersect in a singular curve with a cusp (case 15), and the arrangement is connected with the one in case $25(\mathcal{B}$ internally touches $\mathcal{A}$ at a double conic).

Proof. Note that the index jump across each $3 \times 3$ Jordan block is 1 . Then the only two possible index sequences are derived.

If the index sequence is $\left\langle\hat{1} \varkappa_{+} 2 \mid 3\right\rangle$, by Lemma 1.4 the two ellipsoids have canonical forms

$A=\left(\begin{array}{ccccc} & \varepsilon_{0} & \\ & \varepsilon_{0} & & \\ \varepsilon_{0} & & & \\ & & & \varepsilon_{1}\end{array}\right), B=\left(\begin{array}{cccc} & & \varepsilon_{0} \lambda_{0} & \\ & \varepsilon_{0} \lambda_{0} & \varepsilon_{0} & \\ \varepsilon_{0} \lambda_{0} & \varepsilon_{0} & & \\ & & & \varepsilon_{1} \lambda_{1}\end{array}\right)$,

where $\varepsilon_{0}=\varepsilon_{1}=1$. By setting $x=\frac{\bar{x}+\bar{z}}{\sqrt{2}}, z=\frac{\bar{x}-\bar{z}}{\sqrt{2}}$, the two ellipsoids are transformed to

$$
\begin{aligned}
& \mathcal{A}^{\prime}: \bar{x}^{2}+y^{2}-\bar{z}^{2}+w^{2}=0 \\
& \mathcal{B}^{\prime}: \lambda_{0} y^{2}+\lambda_{0} \bar{x}^{2}-\lambda_{0} \bar{z}^{2}+\sqrt{2} y(\bar{x}-\bar{z})+\lambda_{1} w^{2}=0 .
\end{aligned}
$$

Letting $\bar{z}=0$ be the plane at infinity, $\mathcal{A}^{\prime}$ becomes a unit sphere, and $\mathcal{B}^{\prime}$ becomes an ellipsoid with the equation

$$
\lambda_{0} \bar{x}^{2}+\lambda_{0} y^{2}+\sqrt{2} \bar{x} y-\sqrt{2} y+\lambda_{1} w^{2}-\lambda_{0}=0 .
$$

Letting $\bar{x}=\frac{\hat{x}+\hat{y}}{\sqrt{2}}, y=\frac{\hat{x}-\hat{y}}{\sqrt{2}}$, then $\mathcal{A}^{\prime}$ and $\mathcal{B}^{\prime}$ become

$$
\begin{aligned}
& \mathcal{A}^{\prime}: \hat{x}^{2}+\hat{y}^{2}+w^{2}=1 \\
& \mathcal{B}^{\prime}: \frac{\left(x-\frac{1}{2 A^{2}}\right)^{2}}{\frac{C}{A^{2}}}+\frac{\left(y+\frac{1}{2 B^{2}}\right)^{2}}{\frac{C}{B^{2}}}+\frac{w^{2}}{\frac{C}{\lambda_{1}}}=1,
\end{aligned}
$$

where $A=\lambda_{0}+\frac{\sqrt{2}}{2}, B=\lambda_{0}-\frac{\sqrt{2}}{2}, C=\lambda_{0}+\frac{1}{4 A^{2}}+\frac{1}{4 B^{2}}$. By analyzing the intersection curve of $\mathcal{A}^{\prime}$ and $\mathcal{B}^{\prime}$ in a similar way as before we know that $\mathcal{A}$ and $\mathcal{B}$ intersect at a singular curve with a cusp.

If the index sequence is $\left\langle\hat{1} \mid 2 \varkappa_{+} 3\right\rangle$, a similar analysis is performed. Note that these two arrangements differ in connection with other arrangements. Case 14 is connected with case $26\left(A R_{20}\right)$, but not with case $25\left(A R_{19}\right)$; while case 15 is connected with case 25 , but not with case 26 . The proofs of these connections are shown in Theorem 4.12.

Theorem 3.4. Given two ellipsoids $\mathcal{A}$ and $\mathcal{B}$, if their characteristic polynomial has a double root and two other simple real root with Segre characteristic $[(11) 11]_{3}$, then they have the following two possible index sequences and corresponding arrangements:

(1) $\langle\hat{1}|2||2| 3\rangle-\mathcal{A}$ and $\mathcal{B}$ intersect in two planar conics which intersect at two points (case 16);

(2) $\langle\hat{1}|2| 1|| 3\rangle-\mathcal{B}$ penetrates $\mathcal{A}$ and has two components exterior to $\mathcal{A}$ (case 17 );

(3) $\langle\hat{1}|| 3|2| 3\rangle-\mathcal{A}$ penetrates $\mathcal{B}$ and has two components exterior to $\mathcal{B}$ (case 18 );

(4) $\langle\hat{1}|| 1|2| 3\rangle-\mathcal{A}$ contains $\mathcal{B}$ and touches $\mathcal{B}$ at two points (case 19);

(5) $\langle\hat{1}|2| 3|| 3\rangle-\mathcal{B}$ contains $\mathcal{A}$ and touches $\mathcal{A}$ at two points (case 20);

(6) $\langle 1|0| \hat{1}|| 3\rangle-\mathcal{A}$ and $\mathcal{B}$ are separate (case 21);

(7) $\langle\hat{1}|0||2| 3\rangle$ or $\langle\hat{1}|0| 1|| 3\rangle-\mathcal{A}$ contains but does not touch $\mathcal{B}$ (case 22);

(8) $\langle\hat{1}|| 3|4| 3\rangle$ or $\langle\hat{1}|2||4| 3\rangle-\mathcal{B}$ contains but does not touch $\mathcal{A}$ (case 23).

Proof. We suppose that the double root is $\lambda_{0}$ and the other two simple roots are $\lambda_{1}<\lambda_{2}$. We prove only case 16 , and other cases can be analyzed in a similar way.

If the index sequence is $\langle\hat{1}|2||2| 3\rangle$, then $0<\lambda_{1}<\lambda_{0}<\lambda_{2}$. The two ellipsoids have the canonical forms:

$A_{0}=\operatorname{diag}\left(\varepsilon_{1}, \varepsilon_{2}, \varepsilon_{3}, \varepsilon_{4}\right), B_{0}=\operatorname{diag}\left(\varepsilon_{1} \lambda_{1}, \varepsilon_{2} \lambda_{0}, \varepsilon_{3} \lambda_{0}, \varepsilon_{4} \lambda_{2}\right)$,

where $\varepsilon_{1}=1, \varepsilon_{2}=1, \varepsilon_{3}=-1, \varepsilon_{4}=1$. Letting $z=0$ be the plane at infinity, then $\mathcal{A}_{0}$ is a unit sphere and $\mathcal{B}_{0}$ is an ellipsoid with the equation

$$
\frac{\lambda_{1}}{\lambda_{0}} x^{2}+y^{2}+\frac{\lambda_{2}}{\lambda_{0}} w^{2}=1 .
$$

Then the intersection curve of $\mathcal{A}_{0}$ and $\mathcal{B}_{0}$ lies on the pair of planes

$$
w= \pm \sqrt{\frac{\lambda_{0}-\lambda_{1}}{\lambda_{2}-\lambda_{0}}} x .
$$

Hence the intersection curve of the two ellipsoids consists of two conics that are the intersection of the above two planes with the unit sphere, and these two conics intersect with each other at two real points.

TheOREm 3.5. Given two ellipsoids $\mathcal{A}$ and $\mathcal{B}$, if their characteristic polynomial has a triple real root and a simple real root with Segre characteristic $[(111) 1]_{2}$, then they have the following two possible index sequences and corresponding arrangements:

(1) $\langle\hat{1}|||2| 3\rangle-\mathcal{B}$ internally touches $\mathcal{A}$ at a double conic (case 25);

(2) $\langle\hat{1}|2||| 3\rangle-\mathcal{A}$ internally touches $\mathcal{B}$ at a double conic (case 26);

(3) $\langle\hat{1}|0||| 3\rangle-\mathcal{A}$ contains but does not touch $\mathcal{B}($ case 27$)$;

(4) $\langle\hat{1}|||4| 3\rangle-\mathcal{B}$ contains but does not touch $\mathcal{A}($ case 28$)$;

Proof. We suppose that the triple root is $\lambda_{0}$ and the simple roots is $\lambda_{1}$. We provide a proof for case 25 , and other cases can be similarly analyzed. 
If the index sequence is $\langle\hat{1}|||2| 3\rangle$, then $0<\lambda_{0}<\lambda_{1}$. The canonical forms of $\mathcal{A}$ and $\mathcal{B}$ are

$$
A_{0}=\operatorname{diag}\left(\varepsilon_{1}, \varepsilon_{2}, \varepsilon_{3}, \varepsilon_{4}\right), B_{0}=\operatorname{diag}\left(\varepsilon_{1} \lambda_{0}, \varepsilon_{2} \lambda_{0}, \varepsilon_{3} \lambda_{0}, \varepsilon_{4} \lambda_{1}\right),
$$

where $\varepsilon_{4}=1$ and we set $\varepsilon_{1}=\varepsilon_{2}=1, \varepsilon_{3}=-1$. Letting $z=0$ be the plane at infinity, $\mathcal{A}_{0}$ is a sphere and $\mathcal{B}_{0}$ is the ellipsoid with the equation

$$
x^{2}+y^{2}+\frac{\lambda_{1}}{\lambda_{0}} w^{2}=1 .
$$

Clearly, $\mathcal{B}_{0}$ is inside $\mathcal{A}_{0}$ and touches $\mathcal{A}_{0}$ at the circle which is the intersection of the cylinder $y^{2}+z^{2}=0$ and the plane $x=0$.

Theorem 3.6. Given two ellipsoids $\mathcal{A}$ and $\mathcal{B}$, if their characteristic polynomial has a triple real root and a simple real root with Segre characteristic $[(21) 1]_{2}$, then they have the following two possible index sequences and corresponding arrangements:

(1) $\left\langle\hat{1}\left|2 \chi_{+}\right| 3\right\rangle-\mathcal{B}$ is divided by $\mathcal{A}$ into three parts, and the intersection curve has a crunode (case 29);

(2) $\left\langle\hat{1} \chi_{-}|2| 3\right\rangle-\mathcal{A}$ is divided by $\mathcal{B}$ into three parts, and the intersection curve has a crunode (case 30);

(3) $\left\langle\hat{1} \imath_{+}|2| 3\right\rangle-\mathcal{B}$ internally touches $\mathcal{A}$ at an acnode(case $31)$;

(4) $\left\langle\hat{1}\left|2 \imath_{-}\right| 3\right\rangle-\mathcal{A}$ internally touches $\mathcal{B}$ at an acnode (case 32).

Proof. We shall provide a proof for case 29, and other cases can be similarly analyzed. We suppose that the triple root is $\lambda_{0}$ and the simple real root is $\lambda_{1}$.

If the index sequence is $\left\langle\hat{1}\left|2 \imath_{+}\right| 3\right\rangle$, then $0<\lambda_{1}<\lambda_{0}$, and the two ellipsoids have canonical forms

$A_{0}=\left(\begin{array}{cccc}\varepsilon_{1} & & & \\ & & \varepsilon_{0} & \\ & \varepsilon_{0} & & \\ & & & \varepsilon_{2}\end{array}\right), B_{0}=\left(\begin{array}{cccc}\varepsilon_{1} \lambda_{1} & & & \\ & & \varepsilon_{0} \lambda_{0} & \\ & \varepsilon_{0} \lambda_{0} & \varepsilon_{0} & \\ & & & \varepsilon_{2} \lambda_{0}\end{array}\right)$

where $\varepsilon_{1}=\varepsilon_{0}=\varepsilon_{2}=1$. By setting

$$
y=\frac{\bar{y}+\bar{z}}{\sqrt{2}}, z=\frac{\bar{y}-\bar{z}}{\sqrt{2}},
$$

the equations of $\mathcal{A}$ and $\mathcal{B}$ are transformed to

$$
\begin{aligned}
& \mathcal{A}^{\prime}=x^{2}+\bar{y}^{2}-\bar{z}^{2}+w^{2}=0 \\
& \mathcal{B}^{\prime}=\lambda_{1} x^{2}+\left(\lambda_{0}+\frac{1}{2}\right) \bar{y}^{2}+\left(\lambda_{0}-\frac{1}{2}\right) \bar{z}^{2}+\lambda_{0} w^{2}-\bar{y} \bar{z}=0 .
\end{aligned}
$$

Choose $\bar{z}=0$ as the plane at infinity. Then $\mathcal{A}^{\prime}$ is a unit sphere and $\mathcal{B}^{\prime}$ has the equation

$$
\frac{\lambda_{1}}{\lambda_{0}+\frac{1}{2}} x^{2}+\left(\bar{y}-\frac{1}{2 \lambda_{0}+1}\right)^{2}+\frac{\lambda_{0}}{\lambda_{0}+\frac{1}{2}} w^{2}=\frac{\lambda_{0}^{2}}{\left(\lambda_{0}+\frac{1}{2}\right)^{2}} .
$$

On the plane $x=0$, the section of $\mathcal{A}^{\prime}$ is a unit circle and the section of $\mathcal{B}^{\prime}$ is the ellipse

$$
\frac{\left(\bar{y}-y_{0}\right)^{2}}{a^{2}}+\frac{w^{2}}{b^{2}}=1,
$$

where $y_{0}=\frac{1}{2\left(\lambda_{0}+\frac{1}{2}\right)}, a=\frac{\lambda_{0}}{\lambda_{0}+\frac{1}{2}}, b=\frac{\lambda_{0}}{\sqrt{\left(\lambda_{0}+\frac{1}{2}\right) \lambda_{0}}}$. Note that $y_{0}+a=1$ and $a<1$. The curvature of the ellipsoid $\mathcal{B}^{\prime}$ at the intersection point $\bar{y}=1, w=0$ is $\frac{a}{b^{2}}=1$. Therefore, the intersection curve has a crunode at $(x=0, \bar{y}=1, w=0)$, and $\mathcal{B}$ is separated by $\mathcal{A}$ into three parts.

TheOREM 3.7. Given two ellipsoids $\mathcal{A}$ and $\mathcal{B}$, if their characteristic polynomial has two double roots with Segre characteristic $[2(11)]_{2}$, then they have the following two possible index sequences and corresponding arrangement:

(1) $\left\langle\hat{1} \| 3 \imath_{+} 3\right\rangle-$ part of $\mathcal{A}$ goes inside $\mathcal{B}$ and touches $\mathcal{B}$ internally at an acnode (case 33);

(2) $\left\langle\hat{1} \chi_{-} 1 \| 3\right\rangle-$ part of $\mathcal{B}$ goes inside $\mathcal{A}$ and touches $\mathcal{A}$ internally at an acnode (case 34);

(3) $\left\langle\hat{1} \chi_{+} 1 \| 3\right\rangle-\mathcal{B}$ is inside $\mathcal{A}$ and touches $\mathcal{A}$ at an acnode (case 35);

(4) $\left\langle\hat{1}|| 3 \imath_{-} 3\right\rangle-\mathcal{A}$ is inside $\mathcal{B}$ and touches $\mathcal{B}$ at an acnode (case 36);

(5) $\left\langle 1 \imath_{ \pm} \hat{1}|| 3\right\rangle-\mathcal{A}$ and $\mathcal{B}$ externally touch at an acnode (case 37).

Proof. The proofs for (1)-(4) are very similar to that of Theorem 3.2 , by considering $\lambda_{1}=\lambda_{2}$. For (5), the characteristic polynomial has a double negative root, which means that the two ellipsoids touch externally at an acnode.

Theorem 3.8. Given two ellipsoids $\mathcal{A}$ and $\mathcal{B}$, if their characteristic polynomial has a root of multiplicity four with Segre characteristic $[(31)]_{1}$, then they have only one possible index sequence and corresponding arrangement:

(1) $\left\langle\hat{1} u_{+} \mid 3\right\rangle-\mathcal{A}$ and $\mathcal{B}$ intersect at a conic with an acnode on it (case 38).

Proof. The exchange of the positions of $\mathcal{A}$ and $\mathcal{B}$ does not affect the arrangement, hence the arrangement is determined by the intersection curve. By [Tu et al. 2009], the intersection curve is a conic with an acnode on it.

Theorem 3.9. Given two ellipsoids $\mathcal{A}$ and $\mathcal{B}$, if their characteristic polynomial has two double roots with Segre characteristic $[(11)(11)]_{2}$, then they have the following two possible index sequences and corresponding arrangements:

(1) $\langle\hat{1}|| 1|| 3\rangle-\mathcal{A}$ contains $\mathcal{B}$ and touches $\mathcal{B}$ at two points (case 39);

(2) $\langle\hat{1}|| 3|| 3\rangle-\mathcal{B}$ contains $\mathcal{A}$ and touches $\mathcal{A}$ at two points (case 40);

Proof. The proof is very similar to that of Theorem 3.4, by considering $\lambda_{1}=\lambda_{2}$.

TheOREM 3.10. Given two ellipsoids $\mathcal{A}$ and $\mathcal{B}$, if their characteristic polynomial has a root of multiplicity four with Segre characteristic $[(211)]_{1}$, then they have the following two possible index sequences and corresponding arrangements:

(1) $\left\langle\hat{1} \imath_{+} \| 3\right\rangle-\mathcal{A}$ contains $\mathcal{B}$ and touches $\mathcal{B}$ at two points (case 41);

(2) $\left\langle\hat{1} \imath_{-} \| 3\right\rangle-\mathcal{B}$ contains $\mathcal{A}$ and touches $\mathcal{A}$ at two points (case 42). 
Proof. The proof is similar to that of Theorem 3.2. If the index sequence is $\left\langle\hat{1} \imath_{+} \| 3\right\rangle$, the proof is similar to that of (5) in Theorem 3.2 by letting $\lambda_{0}=\lambda_{1}=\lambda_{2}$. The other cases can be proved similarly.

\section{PROOF OF THE CONNECTION DIAGRAM}

In this section we prove the three stratification diagram in Fig. 12-Fig.14 in the main paper that show the connections of the arrangements.

Note that two modified index sequences with the same degenerate degree are not connected, for example, $\mathbf{a}=\langle\hat{1}|0| 1|2| 3\rangle$ and $\mathbf{b}=\langle\hat{1}|2| 3|4| 3\rangle$ are not connected.

Similar reasons help us to exclude the connections in the following situations. Suppose that $\mathbf{a}=\left\langle\hat{e_{1}}\left|e_{2}\right| e_{3}\left|e_{4}\right| e_{5}\right\rangle$ and $\mathbf{b}=\left\langle\hat{m}_{1} \imath_{-} m_{2}\left|m_{3}\right| m_{4}\right\rangle$ are with neighboring degenerate degrees. If the integer sequence $m_{1}, m_{2}, m_{3}, m_{4}$ is not a subsequence of $e_{1}, e_{2}, e_{3}, e_{4}, e_{5}$, then $\mathbf{a}$ is not connected to $\mathbf{b}$. For example, $\mathbf{a}=\langle\hat{1}|2| 3|2| 3\rangle$ is not connected to $\mathbf{b}=\left\langle\hat{1} \imath_{-} 1|2| 3\right\rangle$, but is possibly connected to $\mathbf{b}^{\prime}=\left\langle\hat{1}\left|2 \ell_{-} 2\right| 3\right\rangle$. We need only to carefully prove the connection between $\mathbf{a}$ and $\mathbf{b}^{\prime}$. Similarly, for $\mathbf{a}=\left\langle\hat{e}_{1}\left|e_{2}\right| e_{3}\right\rangle$ and $\mathbf{b}=\left\langle\hat{m}_{1} \imath_{-} m_{2}\left|m_{3}\right| m_{4}\right\rangle$, if the integer sequence $e_{1}, e_{2}, e_{3}$ is not a subsequence of $m_{1}, m_{2}, m_{3}, m_{4}$, then $\mathbf{a}$ and $\mathbf{b}$ are not connected. On the other hand, the position of the hat $\hat{.}$ is important. For example, $\mathbf{a}=\langle\hat{1}|0| 1|2| 3\rangle$ is possibly connected to $\mathbf{a}=\left\langle\hat{1} \imath_{+} 1|2| 3\right\rangle$ but is not connected to $\mathbf{a}=\left\langle 1 \imath_{-} \hat{1}|2| 3\right\rangle$, where the position of $\hat{\imath}$ is changed.

The exclusion of the above connections can be easily seen by comparing the eigenvalue curves corresponding to the two modifies index sequences, where a small perturbation of one eigenvalue curve can never reach the other curve. By excluding these obvious negative cases, we next prove only the connections between those possible modified index sequence pairs with neighboring degenerate degrees.

Theorem 4.1. $\langle\hat{1}|2| 3\rangle$ is connected to $\left\langle\hat{1}|2| 3 \imath_{ \pm} 3\right\rangle$, i.e., $A R_{6} \sim$ $A R_{9}$ and $A R_{6} \sim A R_{12}$.

Proof. We first prove that $\langle\hat{1}|2| 3\rangle$ is connected to $\left\langle\hat{1}|2| 3 \imath_{-} 3\right\rangle$. Consider the quadratic pair with

$A(t)=\left(\begin{array}{cccc}1 & & & \\ & 1 & & \\ & & & 1 \\ & & 1 & \end{array}\right), B(t)=\left(\begin{array}{cccc}\lambda_{1} & & & \\ & \lambda_{2} & & \\ & & \beta-k t & \alpha \\ & & \alpha & -\beta+t\end{array}\right)$,

where $0<\lambda_{1}<\lambda_{2}<\alpha, 0<\beta$, and $k>1$ be a parameter. Then

$$
A(t)^{-1} B(t)=\left(\begin{array}{cccc}
\lambda_{1} & & & \\
& \lambda_{2} & & \\
& & \alpha & -\beta+t \\
& & \beta-k t & \alpha
\end{array}\right) .
$$

Then clearly at $t=0$, the quadric pair has the index sequence $\langle\hat{1}|2| 3\rangle$, and the roots of the characteristic polynomial are two real roots $\lambda_{1}, \lambda_{2}$ and a pair of conjugate complex roots $\alpha \pm \beta_{i}$ with $\beta>0$.

Let us examine the four eigenvalues of $A(t)^{-1} B(t)$ when $t \neq 0$. Besides the two real eigenvalues $\lambda_{1}, \lambda_{2}$, there are two

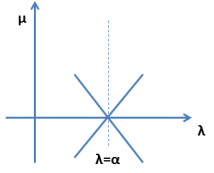

(a) $\varepsilon=0$

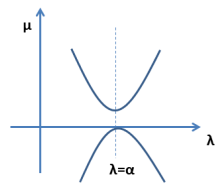

(b) $\varepsilon=-1$

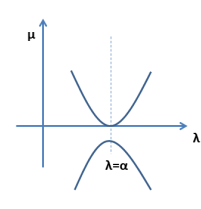

(c) $\varepsilon=+1$
Fig. 3. Local image of the eigenvalue curve of around a double root in $\lambda$ with the Jordan form with $\varepsilon=0,-1,+1$.

other eigenvalues

$$
\lambda_{3,4}=\alpha \pm \sqrt{-\beta^{2}+\beta t+k t \beta-k t^{2}},
$$

which can be complex or real, depending on the value of $t$. Noticing that $k>1$, when $t<\frac{\beta}{k}$ or $t>\beta$, the two roots are a pair of conjugate complex numbers, and the Jordan form of $A(t)^{-1} B(t)$ is

$$
\left(\begin{array}{cccc}
\lambda_{1} & & & \\
& \lambda_{2} & & \\
& & -\sqrt{\beta^{2}-\beta t-k t \beta+k t^{2}} & \sqrt{\beta^{2}-\beta t-k t \beta+k t^{2}}
\end{array}\right)
$$

and the modified index sequence is $\langle\hat{1}|2| 3\rangle$. When $t=\frac{\beta}{k}$ or $t=\beta, \lambda_{3}=\lambda_{4}=\alpha$ creates a double real root, whose corresponding Jordan block has the form

$$
\left(\begin{array}{cccc}
\lambda_{1} & & & \\
& \lambda_{2} & & \\
& & \alpha & \varepsilon \\
& & & \alpha
\end{array}\right)
$$

where $\varepsilon$ can be $0,+1,-1$. Under the assumption that $k>1$, we claim that $\varepsilon=-1$. This is derived by analyzing the image of the eigenvalue curve

$$
C(\lambda, \mu)=\operatorname{det}(\lambda A(t)-B(t)-\mu I)=0
$$

in a small neighborhood $\Gamma$ of the crossing point of the line $\lambda=\alpha$ and $\mu=0$, see Figure 3 for illustration. Let $\mu_{0}>0$ be a sufficiently small number, then $C\left(\lambda, \mu_{0}\right)=0$ has two roots in $\lambda$ in the region $\Gamma$ :

$k \alpha+\frac{\sqrt{-\mu k^{2} \beta+\mu k \beta+\mu^{2} k^{2}}}{k},-k \alpha+\frac{\sqrt{-\mu k^{2} \beta+\mu k \beta+\mu^{2} k^{2}}}{k}$.

Since $\beta>0, \mu_{0}>0$ and $k>1$, these two roots are both complex. Hence the images of different Jordan forms shown in Fig. 3 tell that $\varepsilon=+1$. That is, the modified sequence is $\left\langle\hat{1}|2| 3 \imath_{-} 3\right\rangle$ at $t=\frac{\beta}{k}$ or $t=\beta$. Hence the connection between $\langle\hat{1}|2| 3\rangle$ and $\left\langle\hat{1}|2| 3 \imath_{\_} 3\right\rangle$ is proved.

The connection between $\langle\hat{1}|2| 3\rangle$ and $\left\langle\hat{1}|2| 3 \imath_{+} 3\right\rangle$ can be similarly proved by letting $0<k<1$.

Corollary 4.2. $A R_{6} \sim A R_{7}, A R_{6} \sim A R_{8}, A R_{6} \sim A R_{10}, A R_{6} \sim$ $A R_{11}, A R_{6} \sim A R_{13}$.

Proof. Similar arguments to Theorem 4.1 derive the connections. 
THEOREM 4.3. $\langle\hat{1}|2| 3|2| 3\rangle$ is connected to $\left\langle\hat{1}|2| 3 \imath_{+} 3\right\rangle$, i.e., $A R_{5} \sim A R_{9} ;\langle\hat{1}|2| 3|4| 3\rangle$ is connected to $\left\langle\hat{1}|2| 3 \imath_{-} 3\right\rangle$, i.e., $A R_{3} \sim A R_{12}$.

Proof. For $\langle\hat{1}|2| 3|2| 3\rangle$, the signs of the Jordan forms are $\varepsilon_{1}=$ $\varepsilon_{2}=\varepsilon_{4}=1, \varepsilon_{3}=-1 ;$ for $\langle\hat{1}|2| 3|4| 3\rangle, \varepsilon_{1}=\varepsilon_{2}=\varepsilon_{3}=1, \varepsilon_{4}=$ -1 . Consider the quadratic pair with

$$
\begin{gathered}
A(t)=\operatorname{diag}\left(\varepsilon_{1}, \varepsilon_{2}, \varepsilon_{3}, \varepsilon_{4}-t\right), \\
B(t)=\left(\begin{array}{cccc}
\varepsilon_{1} \lambda_{1} & & \\
& \varepsilon_{2} \lambda_{2} & & \\
& & \varepsilon_{3} \lambda_{3} & t \\
& & & \varepsilon_{4} \lambda_{4}
\end{array}\right) .
\end{gathered}
$$

Then

$$
A(t)^{-1} B(t)=\left(\begin{array}{cccc}
\lambda_{1} & & & \\
& \lambda_{2} & & \\
& & \lambda_{3} & t / \varepsilon_{3} \\
& & & \varepsilon_{4} \lambda_{4} /\left(\varepsilon_{4}-t\right)
\end{array}\right) .
$$

If $\varepsilon_{3}=-1, \varepsilon_{4}=+1$, then within $1-\frac{\lambda_{4}}{\lambda_{3}}<t<1$, the matrix $A(t)^{-1} B(t)$ has the Jordan form

$$
\left(\begin{array}{llll}
\lambda_{1} & & & \\
& \lambda_{2} & & \\
& & \lambda_{3} & \\
& & & \lambda_{4} /(1-t)
\end{array}\right)
$$

while at $t=1-\frac{\lambda_{4}}{\lambda_{3}}$, the Jordan form of $A(t)^{-1} B(t)$ suddenly changes to

$$
\left(\begin{array}{cccc}
\lambda_{1} & & & \\
& \lambda_{2} & & \\
& & \lambda_{3} & 1 \\
& & & \lambda_{3}
\end{array}\right)
$$

This proves the connection $A R_{5} \sim A R_{9}$. For the case $\varepsilon_{3}=$ $+1, \varepsilon_{4}=-1$, we reset

$$
A(t)=\operatorname{diag}\left(\varepsilon_{1}, \varepsilon_{2}, \varepsilon_{3}-t, \varepsilon_{4}\right) .
$$

Then within $1-\frac{\lambda_{3}}{\lambda_{4}}<t<1$, the matrix $A(t)^{-1} B(t)$ has the Jordan form

$$
\left(\begin{array}{llll}
\lambda_{1} & & & \\
& \lambda_{2} & & \\
& & \lambda_{3} /(1-t) & \\
& & & \lambda_{4}
\end{array}\right),
$$

while at $t=1-\frac{\lambda_{3}}{\lambda_{4}}$, the Jordan form of $A(t)^{-1} B(t)$ suddenly changes to

$$
\left(\begin{array}{cccc}
\lambda_{1} & & & \\
& \lambda_{2} & & \\
& & \lambda_{4} & 1 \\
& & & \lambda_{4}
\end{array}\right)
$$

This proves the connection $A R_{3} \sim A R_{12}$.
TheOrEm 4.5. $\left\langle\hat{1}|2| 3 \imath_{+} 3\right\rangle$ is connected to $\left\langle\hat{1} \mid 2 \varkappa_{+} 3\right\rangle$, i.e., $A R_{9} \sim A R_{14}$.

Proof. Let the two moving quadrics be

$$
A(t)=\left(\begin{array}{cccc}
1 & & & \\
& & & 1 \\
& & 1 & \\
& 1 & &
\end{array}\right), B(t)=\left(\begin{array}{cccc}
1 & & & \\
& & 2-t & 1 \\
& 2 & 1 & 0
\end{array}\right) .
$$

Then

$$
A(t)^{-1} B(t)=\left(\begin{array}{cccc}
1 & & & \\
& 2 & 1 & \\
& & 2-t & 1 \\
& & & 2
\end{array}\right) .
$$

When $t=0$, the Jordan form of $A(t)^{-1} B(t)$ is

$$
J\left(A(0)^{-1} B(0)\right)=\left(\begin{array}{cccc}
1 & & & \\
& 2 & 1 & \\
& & 2 & 1 \\
& & & 2
\end{array}\right) .
$$

When $0<t<1$, the Jordan form of $A(t)^{-1} B(t)$ is

$$
J\left(A(t)^{-1} B(t)\right)=\left(\begin{array}{cccc}
1 & & & \\
& 2-t & & \\
& & 2 & 1 \\
& & & 2
\end{array}\right) .
$$

This proves the connection.

Corollary 4.6. $A R_{8} \leftrightarrow A R_{14}, A R_{7} \leftrightarrow A R_{15}, A R_{10} \leftrightarrow$ $A R_{15}$.

Proof. The proof is similar to that of Theorem 4.5.

THEOREM 4.7. $\left\langle\hat{1} \imath_{ \pm} 1|2| 3\right\rangle$ is connected to $\langle 1|| 1|2| 3\rangle$, i.e., $A R_{10} \sim A R_{17}, A R_{11} \sim A R_{17}$.

Proof. Suppose that the sign of the Jordan forms are $\varepsilon_{1}=$ $\varepsilon_{2}=\varepsilon_{3}=1$. Consider the moving quadric pair with

$A=\left(\begin{array}{cccc} & \varepsilon_{1} & & \\ \varepsilon_{1} & & & \\ & & \varepsilon_{2} & \\ & & & \varepsilon_{3}\end{array}\right), B(t)=\left(\begin{array}{cccc} & \varepsilon_{1} \lambda_{1} & & \\ \varepsilon_{1} \lambda_{1} & \varepsilon_{1} t & & \\ & & \varepsilon_{2} \lambda_{2} & \\ & & & \varepsilon_{3} \lambda_{3}\end{array}\right)$,

where $\lambda_{1}<\lambda_{2}<\lambda_{3}$ are the real roots of the characteristic polynomial, with $\lambda_{1}$ being a double one. Then

$$
A^{-1} B(t)=\left(\begin{array}{cccc}
\lambda_{1} & t & & \\
& \lambda_{1} & & \\
& & \lambda_{2} & \\
& & & \lambda_{3}
\end{array}\right) .
$$

Clearly, when $t=0$, the modified index sequence is $\langle 1|| 1|2| 3\rangle$, while when $t<0$, the modified index sequence is $\left\langle\hat{1} \imath_{+}+1|2| 3\right\rangle$. Hence this proves the connection $A R_{10} \sim A R_{17}$.

The other case when $\varepsilon_{1}=-1$ can be similarly derived.

Corollary 4.8. $A R_{9} \sim A R_{18}, A R_{12} \sim A R_{18}, A R_{8} \sim$

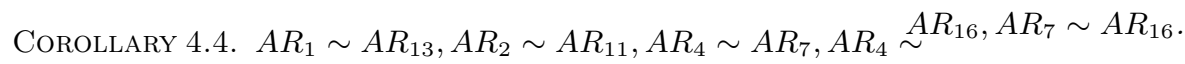
$A R_{10}, A R_{5} \sim A R_{8}$.

Proof. The proof is similar to that of Theorem 4.7.

Proof. Similar arguments to Theorem 4.3 derive the connections.
TheOREM 4.9. $\langle 1|2||2| 3\rangle$ is connected to $\langle 1|2||| 3\rangle$, i.e., $A R_{16} \sim$ $A R_{20}$. 
Proof. We show only $A R_{16} \sim A R_{20}$, other cases can be similarly derived. Consider the two moving quadrics

$A=\left(\begin{array}{cccc}1 & & & \\ & 1 & & \\ & & -1 & \\ & & & 1\end{array}\right), B=\left(\begin{array}{cccc}\lambda_{1} & & & \\ & \lambda_{2} & & \\ & & -\lambda_{2} & \\ & & & \lambda_{3}-t\end{array}\right)$,

where $\lambda_{1}<\lambda_{2}<\lambda_{3}$. Then when $\lambda_{2}<t<\lambda_{3}-\lambda_{2}$, the modified index sequence is $\langle 1|2||2| 3\rangle$, and when $t=\lambda_{3}-$ $\lambda_{2}$, the modified index sequence is $\langle 1|2||| 3\rangle$. This proves the connection.

Corollary 4.10. $A R_{16} \sim A R_{19}, A R_{18} \sim A R_{20}, A R_{17} \sim$ $A R_{19}$.

Proof. The connections can be proved similarly to Theorem 4.9 .

Corollary 4.11. $\left\langle 1 \mid 2 \chi_{+} 3\right\rangle$ is connected to $\langle 1|2||| 3\rangle$, i.e., $A R_{14} \sim A R_{20}, A R_{15} \sim A R_{19}$.

Proof. Consider the moving quadric pair

$$
A=\left(\begin{array}{cccc}
1 & & & \\
& & & 1 \\
& & 1 & \\
& 1 & &
\end{array}\right), B=\left(\begin{array}{ccccc}
\lambda_{1} & & & \\
& & & \lambda_{2} \\
& & \lambda_{2} & t \\
& \lambda_{2} & t &
\end{array}\right)
$$

with $0<\lambda_{1}<\lambda_{2}$. Then

$$
A^{-1} B(t)=\left(\begin{array}{cccc}
\lambda_{1} & & & \\
& & & \lambda_{2} \\
& & \lambda_{2} & t \\
& \lambda_{2} & t &
\end{array}\right)
$$

The modified index sequence is $\langle 1|2||| 3\rangle$ when $t=0$, and is $\left\langle 1 \mid 2 \imath_{+} 3\right\rangle$ when $t>0$. Hence $A R_{14} \sim A R_{20}$. Similarly we can prove that $A R_{15} \sim A R_{19}$.

Theorem 4.12. $A R_{14} \sim A R_{20}, A R_{15} \sim A R_{21}$.

Proof. Consider the moving quadric pair

$$
A=\left(\begin{array}{ccccc}
1 & & & \\
& & & 1 \\
& & 1 & \\
& 1 & &
\end{array}\right), B=\left(\begin{array}{cccc}
\lambda_{1}+t & & & \\
& & & \lambda_{2} \\
& & \lambda_{2} & 1 \\
& \lambda_{2} & 1 &
\end{array}\right)
$$

with $0<\lambda_{1}<\lambda_{2}$. Then

$$
A^{-1} B(t)=\left(\begin{array}{cccc}
\lambda_{1}+t & & & \\
& & & \lambda_{2} \\
& & \lambda_{2} & 1 \\
& \lambda_{2} & 1 &
\end{array}\right) .
$$

The modified index sequence is $\left\langle 1 \imath_{+} \mid 3\right\rangle$ when $t=\lambda_{2}-\lambda_{1}$, and is $\left\langle 1 \mid 2 \imath_{+} 3\right\rangle$ when $0<t<\lambda_{2}-\lambda_{1}$. Hence $A R_{14} \sim A R_{21}$. Similarly we can prove that $A R_{15} \sim A R_{21}$.

\section{REFERENCES}

C. Tu, W. Wang, B. Mourrain, and J. Wang. 2009. Using signature Sequence to classify intersection curves of two quadrics. Computer Aided Geometric Design 26, 3 (2009), 317-335.

F. Uhlig. 1976. A canonical form for a pair of real symmetric matrices that generate a nonsingular pencil. Linear Algebra Appl. 14, 3 (1976), 189 - 209. https://doi.org/10.1016/0024-3795(76)90066-5

ACM Transactions on Graphics, Vol. 39, No. 3, Article 27. Publication date: May 2020. 